ARTICLE

https://doi.org/10.1038/s41467-019-13247-6

\title{
Structural determinants of microtubule minus end preference in CAMSAP CKK domains
}

\author{
Joseph Atherton ${ }^{1 \star}$, Yanzhang Luo (10 ${ }^{2}$, Shengqi Xiang ${ }^{2,9}$, Chao Yang ${ }^{3}$, Ankit Rai (1) ${ }^{3}$, Kai Jiang (1) ${ }^{4}$, \\ Marcel Stangier ${ }^{5}$, Annapurna Vemu ${ }^{6}$, Alexander D. Cook ${ }^{1}$, Su Wang ${ }^{1}$, Antonina Roll-Mecak (D) 6,7, \\ Michel O. Steinmetz (10 5,8, Anna Akhmanova (1) ${ }^{3}$, Marc Baldus (iD ${ }^{2 \star} \&$ Carolyn A. Moores (i) ${ }^{1 \star}$
}

CAMSAP/Patronins regulate microtubule minus-end dynamics. Their end specificity is mediated by their CKK domains, which we proposed recognise specific tubulin conformations found at minus ends. To critically test this idea, we compared the human CAMSAP1 CKK domain (HsCKK) with a CKK domain from Naegleria gruberi ( $\mathrm{NgCKK}$ ), which lacks minus-end specificity. Here we report near-atomic cryo-electron microscopy structures of HsCKK- and $\mathrm{NgCKK}$-microtubule complexes, which show that these CKK domains share the same protein fold, bind at the intradimer interprotofilament tubulin junction, but exhibit different footprints on microtubules. NMR experiments show that both $\mathrm{HsCKK}$ and $\mathrm{NgCKK}$ are remarkably rigid. However, whereas $\mathrm{NgCKK}$ binding does not alter the microtubule architecture, HsCKK remodels its microtubule interaction site and changes the underlying polymer structure because the tubulin lattice conformation is not optimal for its binding. Thus, in contrast to many MAPs, the HsCKK domain can differentiate subtly specific tubulin conformations to enable microtubule minus-end recognition.

\footnotetext{
${ }^{1}$ Institute of Structural and Molecular Biology, Birkbeck, University of London, Malet Street, London, UK. ${ }^{2}$ NMR Spectroscopy, Bijvoet Center for Biomolecular Research, Utrecht University, Padualaan 8, 3584 CH Utrecht, The Netherlands. ${ }^{3}$ Cell Biology, Department of Biology, Faculty of Science, Utrecht University, Padualaan 8, 3584 CH Utrecht, The Netherlands. ${ }^{4}$ Medical Research Institute, School of Medicine, Wuhan University, 430071 Wuhan, China. ${ }^{5}$ Laboratory of Biomolecular Research, Division of Biology and Chemistry, Paul Scherrer Institut, Villigen, PSI, Switzerland. ${ }^{6}$ Cell Biology and Biophysics Unit, National Institute of Neurological Disorders and Stroke, Bethesda, MD 20892, USA. ${ }^{7}$ Biophysics Center, National Heart, Lung and Blood Institute, Bethesda, MD 20892, USA. ${ }^{8}$ University of Basel, Biozentrum, CH-4056 Basel, Switzerland. ${ }^{9}$ Present address: MOE Key Lab for biomolecular Condensates \& Cellular Dynamics, School of Life Sciences, University of Science and Technology of China, 96 Jinzhai Road, Hefei 230026 Anhui, China. ${ }^{\star}$ email: j.atherton@mail. cryst.bbk.ac.uk; M.Baldus@uu.nl; c.moores@mail.cryst.bbk.ac.uk
} 
T he involvement of the microtubule (MT) cytoskeleton in numerous processes in eukaryotic cells is enabled by the diverse and adaptable properties of individual MTs. MTs act as tracks for molecular motors, while growing and shrinking MTs can be used to generate force. MTs can also act as signalling hubs, such that specific tubulin conformations within particular regions of the polymer stimulate recruitment of distinct MTbinding partners. The molecular basis of these effects, mediated by the conformational adaptability of tubulin dimers, is only just beginning to be understood and represents a key topic in the cytoskeleton field.

The ends of MTs are important sites of conformational diversity and are often points of communication between the MT cytoskeleton and other cellular components, such as membranes, organelles, centrosomes and chromosomes ${ }^{1}$. The exact conformation(s) of MT ends is an ongoing source of debate, but current evidence points to their being composed of zones with distinct and dynamic tubulin conformations ${ }^{2-7}$. MT minus ends were long thought to be static and structurally homogeneous, capped by $\gamma$-TuRCs and buried at MT organizing centres. More recently, however, the discovery and characterisation of CAMSAP (calmodulin-regulated spectrin-associated proteins)/Patronin family members has revealed that control of non-centrosomal MT minus-end dynamics, and their interaction with specific cellular regions, are vital in numerous aspects of cell physiology 8,9 . CAMSAP/Patronins are centrally involved in diverse activities including promoting cell polarity, regulation of neuronal differentiation and axonal regeneration, and definition of spindle organization and asymmetry, thereby highlighting the importance of regulation of MT minus-end dynamics in these varied contexts ${ }^{10-20}$.

At the molecular level, CAMSAPs/Patronins stabilise uncapped MT minus ends and support MT minus-end growth ${ }^{21-23}$. CAMSAP/Patronins are large, multi-domain proteins with many cellular binding partners. However, the family is defined by the presence of a CKK domain (originally identified in CAMSAP1, KIAA1078 and KIAA1543), which is necessary and sufficient for MT minus-end binding in many CAMSAP/Patronins ${ }^{21,24}$. Previously, we showed that CAMSAP/Patronin CKK domains preferentially bind to a zone behind the extreme MT minus end, which corresponds to a region where the lattice undergoes a transition to gently curved tubulin sheets ${ }^{2}$. Subnanometer resolution single particle cryo-EM showed that CKK domains bind on the MT lattice between two tubulin dimers on adjacent protofilaments. Mutagenesis of residues at the MT-binding interface in the CKK domain disrupted lattice and minus end binding, showing that the same regions of the CKK domain that contact the MT are also involved in binding to the minus-end zone. Taking these data together, we proposed a model for CAMSAP/ Patronin MT minus end recognition, which is mediated by sensitivity of the CKK domain to a curved sheet-like conformation of tubulin exclusive to MT minus ends. Specifically, the model suggested that the tighter CKK interaction with $\beta$-tubulin disfavours binding at MT plus ends while the looser $\alpha$-tubulin contacts preferentially accommodate tubulin curvature at minus ends. This interaction also can occur on the MT lattice, but CKK binding induces distortion of the non-optimal binding site configuration, manifesting as protofilament skew within the polymer.

Despite these findings, several critical questions relating to the structural basis of this recognition mechanism remain unanswered: How is the CKK-induced MT lattice distortion accommodated, and what can this tell us about minus-end recognition? Can CKK binding to different MT protofilament architectures shed further light on the mechanism of minus end recognition? Intriguingly, we also previously identified CKKs in the amoeboflagellate N. gruberi and the potato blight fungus $P$. infestans that lacks the binding preference for MT minus ends that was proposed to be present in CKK domains from the last eukaryotic common ancestor ${ }^{2}$. Can a comparison of CKK domains with and without minus-end binding specificity also provide insight into MT minus-end recognition? Since discrimination between different MT lattice zones can depend on relatively subtle structural differences, high resolution information is needed to address these questions. It is currently not possible to image MT minus ends directly at the necessary resolution to observe these conformational variations. However, our previous work showed that CKK lattice binding can be used as a proxy for minus end binding, and cryo-EM studies of the lattice could yield nearatomic resolution information about the CKK-tubulin complex.

We therefore developed a RELION ${ }^{25-27}$-based imageprocessing pipeline that enabled us to study the CKK-MT interaction at better than $4 \AA$ resolution ${ }^{28}$. Using this, we determined reconstructions of MT-bound CKK domains on both 13and 14-protofilament pseudo-helical MTs, allowing visualisation of a wider repertoire of tubulin conformations. We also investigated the CKK domain from Naegleria gruberi (NgCKK), which does not show minus-end binding preference. The direct comparison of tubulin binding by $\mathrm{NgCKK}$ with that of human CAMSAP1 CKK (HsCKK)-which has minus end binding preference-allowed us to probe our previous model of minus-end recognition. We found that $\mathrm{NgCKK}$, like $\mathrm{HsCKK}$, binds between two tubulin dimers in neighbouring protofilaments. However, $\mathrm{NgCKK}$ MT binding is shifted relative to $\mathrm{HsCKK}$, resulting in a modified interaction with tubulin including even smaller contacts with a-tubulin, contrary to our previous prediction. Thus, the comparison between $\mathrm{NgCKK}$ and $\mathrm{HsCKK}$ shows that looser contacts with $\alpha$-tubulin compared to $\beta$-tubulin are important but not sufficient for minus end recognition. $\mathrm{NgCKK}$ binding does not induce protofilament skew, reinforcing that induction of skew is a structural signature for minus-end binding capability. NMR studies revealed a remarkable structural rigidity of both the HsCKK and NgCKK domains. The ability of HsCKK to remodel MTs thus results from a combination of intrinsic structural properties and the precise mode of interaction with its MTbinding site. HsCKK-induced skew arises within the MT lattice from the tilting of entire protofilaments coincidental with contraction of the MT diameter. These multi-disciplinary data reveal that the surprising structural plasticity of MTs, which is distinct from nucleotide-dependent modulations of the MT lattice, forms the basis for minus-end recognition by CAMSAP proteins.

\section{Results}

Canonical lattice binding by a CKK domain from N. gruberi. The presence of CKK domains in diverse organisms presents a unique resource that can shed light on the conserved or divergent properties of these domains. Our previous analysis suggested that a CKK domain from $N$. gruberi did not exhibit MT minus-end binding preference ${ }^{2}$. We confirmed this using TIRF experiments, showing that, in comparison to the well-characterised MT minusend preference of $\mathrm{HsCKK}$ (Fig. 1a, left panel), NgCKK strongly bound along the entire MT lattice and exhibited no MT minusend preference on dynamic MTs at a range of concentrations (Fig. 1a, right panels).

To investigate this distinctive behaviour further, complexes formed by either $\mathrm{NgCKK}$ or HsCKK and taxol-stabilised MTs were imaged using cryo-EM for structure determination. Our previous work showed that CKK MT binding includes interactions with the C-terminal tails of tubulin (CTTs). To facilitate visualisation of this interaction, we used MTs assembled from tubulin purified from a human tsA201 cell line for our reconstructions ${ }^{29}$. These MTs contain only two $\beta$-tubulin 
a

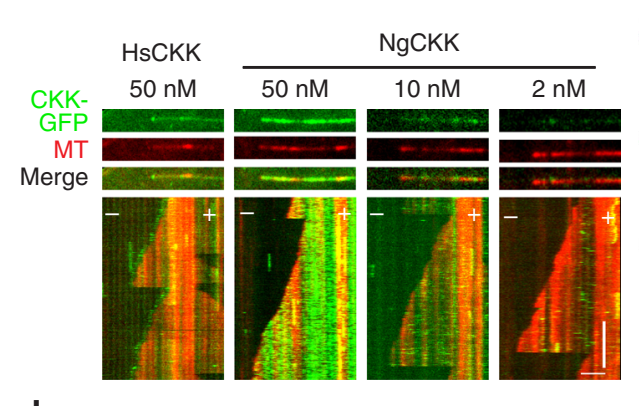

d

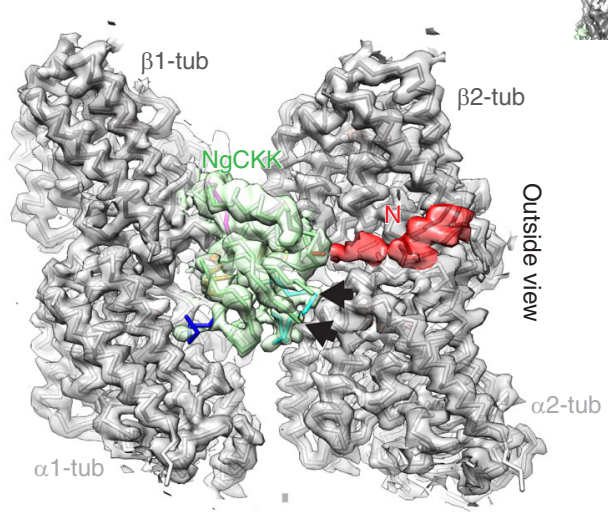

e
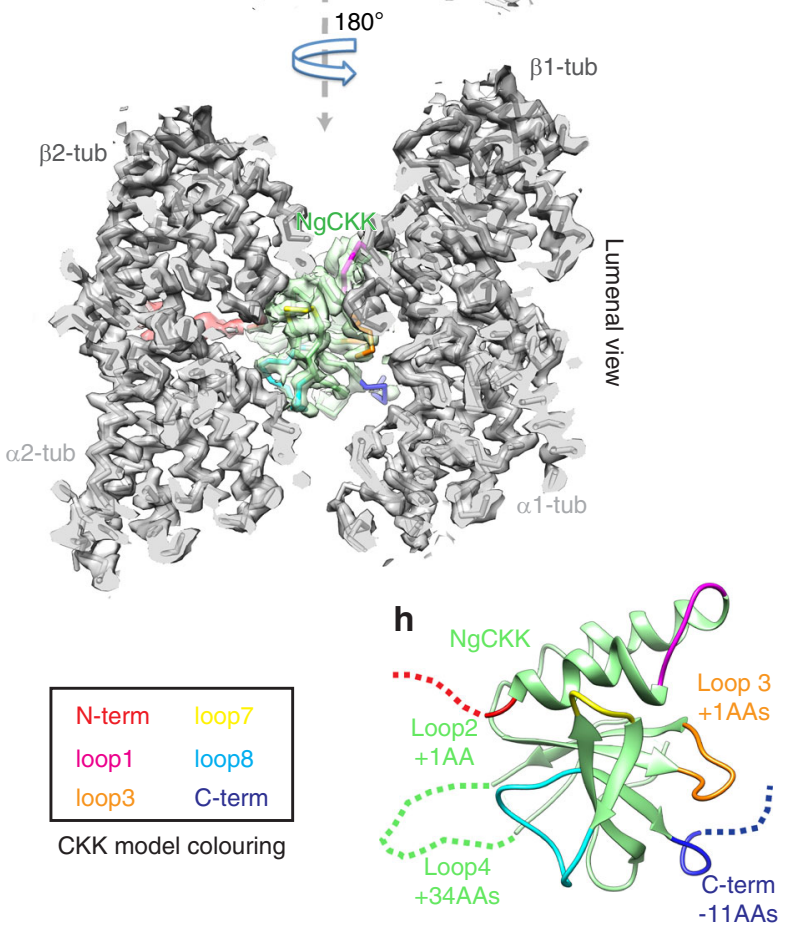

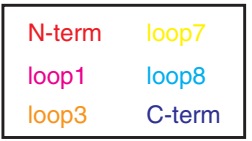

CKK model colouring b
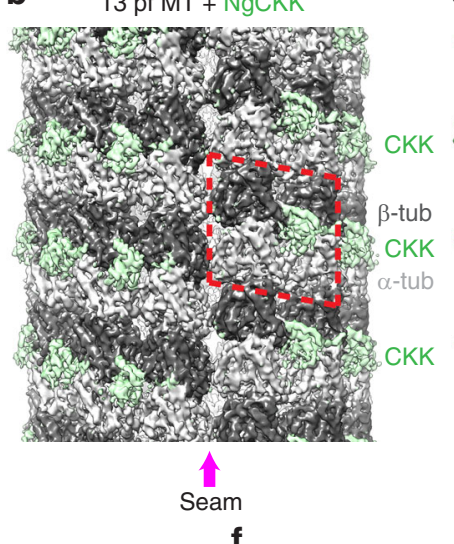

C $\quad 13 \mathrm{pf} \mathrm{MT}+\mathrm{HsCKK}$

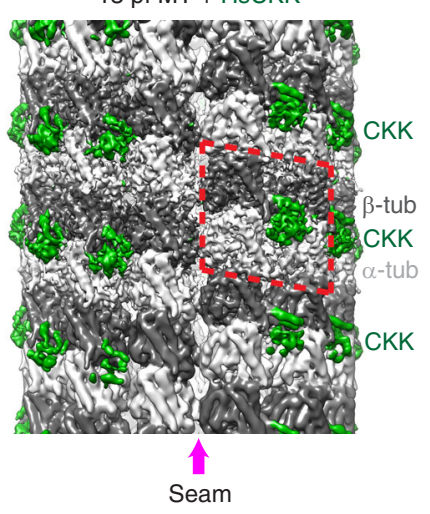

f
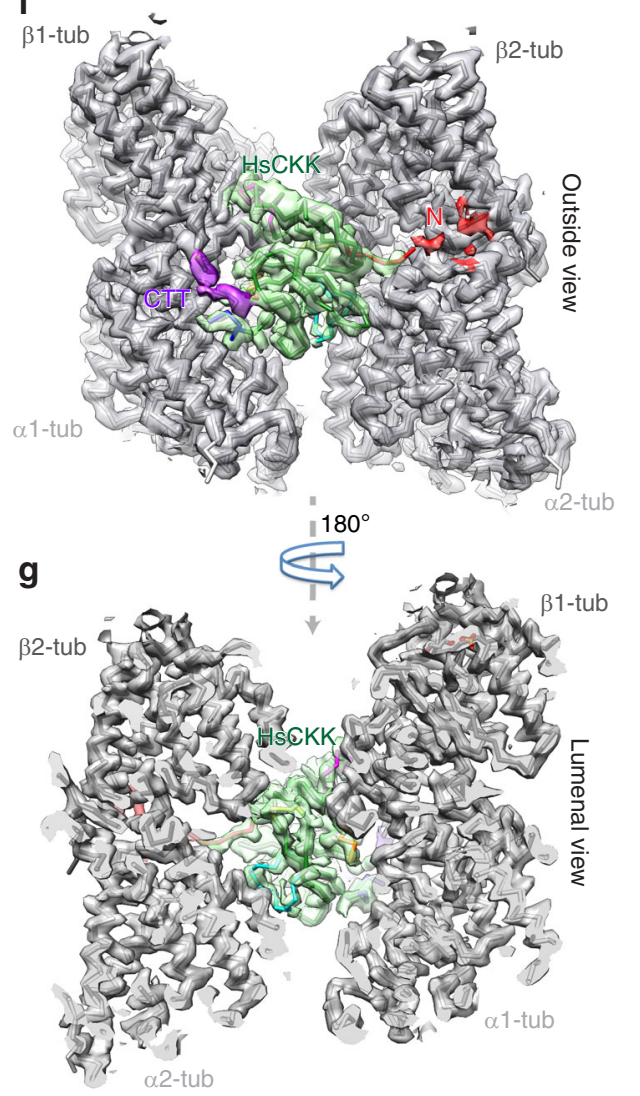

isoforms and one a-tubulin isoform, have no detectable posttranslational modifications as indicated by mass spectrometric analyses ${ }^{29}$, and are thus much more homogenous than the brain tubulin we previously used. To visualise CKK binding at higher resolution, we also developed an image-processing pipeline for pseudo-helical MTs and different protofilament architectures in RELION (see methods in ref. ${ }^{28}$ ). The resulting unsymmetrised (C1) reconstructions for both $\mathrm{NgCKK}$ and $\mathrm{HsCKK}$ showed distinct CKK intradimer, interprotofilament densities every $8 \mathrm{~nm}$ along the MT axis and an absence of CKK density at the seam (Fig. 1b, c). This validates the accuracy of the pipeline and is consistent with our previous work $^{2}$, while revealing the MTbound $\mathrm{NgCKK}$ and $\mathrm{HsCKK}$ complexes at substantially higher resolutions now for both 13- and 14-protofilament MTs. The C1 reconstructions all have resolutions of $4.7 \AA$ or better, and the symmetrised reconstructions have resolutions of $3.8 \AA$ or better (Supplementary Fig. 1). This allowed us to build atomic models of the NgCKK-MT and HsCKK-MT complexes (Fig. 1d-g, Supplementary Fig. 1i-l, Supplementary Table 1).

While the structures of mammalian CKK domains have previously been determined, our NgCKK reconstructions now reveal the near-atomic resolution structure of a non-mammalian CKK domain (Fig. 1h). It has a typical CKK fold, but sequence differences compared to HsCKK (Supplementary Fig. 2a) are reflected in structural differences in several loop regions (Supplementary Fig. 2b). NgCKK's loop4, which faces away from 
Fig. 1 Comparison of the CKK domain from N. gruberi with HsCKK. a Dynamic MT TIRF assays demonstrate that unlike HsCKK, NgCKK does not have a preference for MT minus ends but binds along the whole MT lattice at a range of concentrations. Scale bars: horizontal, $2 \mu \mathrm{m} ;$ vertical, 2 min. $\mathbf{b}$ C1 reconstruction of $\mathrm{NgCKK}$-bound tsA201-tubulin 13-protofilament MT at $4.3 \AA$ resolution, showing CKK density (light green) every $8 \mathrm{~nm}$ between protofilaments at the intradimer tubulin interface, except at the seam; the reconstruction procedure produces a resolution gradient in the density, highest in the middle and lowest at the top/bottom. c C1 reconstruction of HsCKK-bound tsA201-tubulin 13-protofilament MT at 4.5 $\AA$ resolution, showing CKK density (green) bound every $8 \mathrm{~nm}$ between protofilaments at the intradimer tubulin interface, except at the seam; as above, the reconstruction procedure produces a resolution gradient in the density. $\mathbf{d}$ Asymmetric unit extracted from the symmetrized 13-protofilament reconstruction viewed from the outside of the MT reveals the NgCKK-tubulin interaction, including ordering of the CKK N-terminus (red). The CKK model coloured according to CKK SSE/loop colour scheme at the bottom of the figure. Arrows show the start and end of the disordered 34 amino acid loop 4 insert in NgCKK; e $180^{\circ}$ rotated view of panel d, showing the NgCKK interaction from the MT lumen with a cut through of the MT, showing the $\mathrm{NgCKK}$ as a wedge between four tubulin

monomers. f Asymmetric unit extracted from the symmetrized 13-protofilament reconstruction viewed from the outside of the MT reveals the HsCKKtubulin interaction, including partial ordering of the CKK N-terminus (red) and visualization of the unmodified tsA201 cell-tubulin $\beta$-tubulin C-terminal tail (purple). The CKK model coloured according to CKK SSE/loop colour scheme at the bottom of the figure; $\mathbf{g} 180^{\circ}$ rotated view of panel $\mathbf{f}$, showing the CKK interaction from the MT lumen with a cut through the MT, showing the CKK as a wedge between 4 tubulin monomers and exhibiting well-ordered tubulin interaction loops. $\mathbf{h} \mathrm{NgCKK}$ domain with loops coloured, indicating loop insertions and a shortened C-terminus (residue length differences in key loop regions compared to HsCKK are indicated)

the MT surface (Fig. 1d, black arrows), is 34 amino acids longer than in HsCKK. There is no extra density in the cryo-EM reconstructions corresponding to this insert even at low thresholds (Fig. 1d), suggesting it is highly flexible/disordered and unlikely to be involved in MT binding. However, there are also structural differences in regions closer to the MT surface: specifically, loop3, the C-terminal single-turn helix and the beta-hairpin leading into loop7, all show backbone RMSDs $>2.5$ $\AA$ (Supplementary Fig. 2c).

The reconstructions show that both $\mathrm{NgCKK}$ and HsCKK form an intradimer interprotofilament wedge (Fig. 1d-g), contacting both $\alpha$ - and $\beta$-tubulin subunits in a tubulin dimer pair (with constituent monomers numbered $\alpha 1, \beta 1, \alpha 2$ and $\beta 2$ ). Both CKK domains form MT contacts mainly via a set of surface exposed loops (Fig. 1d-g, described below). The N-terminal extension of each CKK domain also contacts the MT but in distinct ways. The $\mathrm{NgCKK} \mathrm{N}$-terminus forms an ordered density that is associated with the surface of $\beta 2$-tubulin, although the density was not sufficiently defined to allow accurate modelling (Fig. 1d). Conversely, the HsCKK N-terminus closest to the CKK core forms a distinct interaction with $\beta 2$-tubulin that was built into the atomic model, whereas density corresponding to its most $\mathrm{N}$ terminal part was hardly visible on the surface of $\beta 2$-tubulin (Fig. 1f). Furthermore, there is no clear contact between NgCKK and the $\beta 1$-tubulin CTT (Fig. 1d). This is in contrast to HsCKK, where density corresponding to an additional $\sim 5$ residues of $\beta$ tubulin's CTT were visualised in our structures (Fig. 1f, purple) contacting both the CKK core and its C-terminus (Supplementary Fig. 3a). The visualisation of $\beta$-tubulin's CTT interaction with HsCKK is likely facilitated by the limited sequence variability (Supplementary Fig. 3b) and lack of post-translational modifications in the CTT tails in tsA201 $\beta$-tubulin compared to brain tubulin 29 .

Overall, these structures show that the core of CKK domains with and without minus-end binding preference have the same protein fold and interact with MTs in similar although not identical ways. Differences in the tubulin interactions are also seen at both their N- and C-termini. Our previous work showed that although these regions contribute to HsCKK MT affinity, neither the $\mathrm{N}$ - nor C-terminal extensions define its minus-end binding specificity ${ }^{2}$. However, to test the hypothesis that the absence of minus-end recognition by $\mathrm{NgCKK}$ is mediated by its termini (i.e. that all $\mathrm{CKK}$ cores have minus end recognition properties), we studied a set of CKK N-/C-termini truncations and chimeras using TIRF microscopy (Supplementary Fig. 4). Removal of either or both termini, and swapping the N-terminus of $\mathrm{NgCKK}$ for that of $\mathrm{HsCKK}$, drastically reduced the overall affinity of these proteins for MTs. Crucially, the chimera in which the C-terminus of $\mathrm{NgCKK}$ was substituted with that of $\mathrm{HsCKK}$ retained MT binding but also showed no minus end recognition, clearly arguing against the above gain-of-function hypothesis. Intriguingly, however, the chimera with both $\mathrm{N}$ - and C-termini of HsCKK grafted onto the $\mathrm{NgCKK}$ core has a slight minus-end preference, albeit significantly less than HsCKK. This hints at modulatory mechanisms of the unstructured termini-in particular the N-terminus-on the core CKK domain. Nevertheless, the behaviour of most of these engineered constructs, together with the observation of similar binding sites for $\mathrm{NgCKK}$ and $\mathrm{HsCKK}$, and with our previous characterisation of $\mathrm{HsCKK}$ mutants, suggests that differences in CKK minus-end recognition behaviours are primarily due to structural differences in the interaction between tubulin and the CKK core. The near-atomic resolution of all our reconstructions allowed us to investigate the mechanistic basis of these effects.

Differences in MT interaction between NgCKK and HsCKK. To visualise how NgCKK's MT interaction differs from HsCKK, each CKK-tubulin model was aligned on the tubulin parts of the complex, thereby revealing differences in CKK positioning relative to the MT lattice (Fig. 2a, top). The tubulin dimers in both models readily superimpose (Supplementary Fig. 5), as do helix$\alpha 1$, loop 1 and loop7 in each CKK domain (Fig. 2a, top). However, NgCKK loop3, loop8 and its C-terminus-which all contact the MT-are displaced compared to HsCKK (Fig. 2a, bottom). While some of these variations are due to structural divergence in $\mathrm{NgCKK}$, this is insufficient to explain all the differences in CKK positioning relative to tubulin. In fact, $\mathrm{NgCKK}$ is rotated away from the MT around the apex of loop7 relative to HsCKK (Supplementary Movie 1).

As a result of this shift, even when the sequences in each CKK domain are conserved, our reconstructions show that some $\mathrm{NgCKK}$ residues engage differently with the MT lattice compared to equivalent residues in HsCKK. For example, HsCKK loop3 residues R1535 and R1528 contribute significantly to MT affinity. With the improved resolution of our current reconstructions, R1528 is observed extending close to a1-tubulin's residues $\mathrm{H} 393$ on H11, whilst R1535 reaches to contact the $\beta 1$-tubulin CTT (Fig. 2b, top). On the other hand, in NgCKK loop3-which is one residue longer and positioned differently with respect to the MT surface compared to $\mathrm{HsCKK}$ - the residue equivalent to $\mathrm{R} 1528$ (R678) interacts with loop H10- $\beta 7$ of $\beta 1$-tubulin, possibly hydrogen bonding with E345 (Fig. 2b, bottom). In addition, instead of interacting with al-tubulin, NgCKK residue R686 
a

a
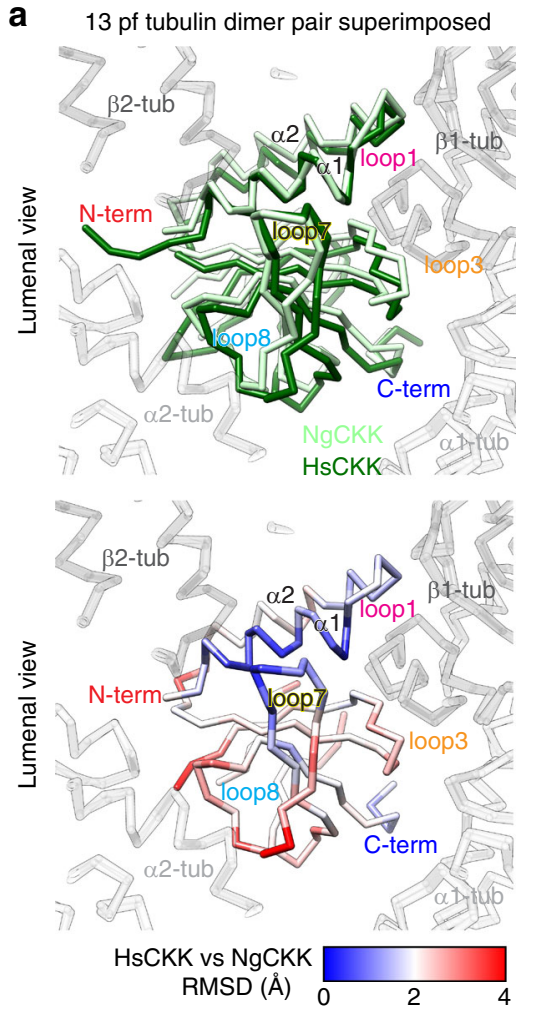

b
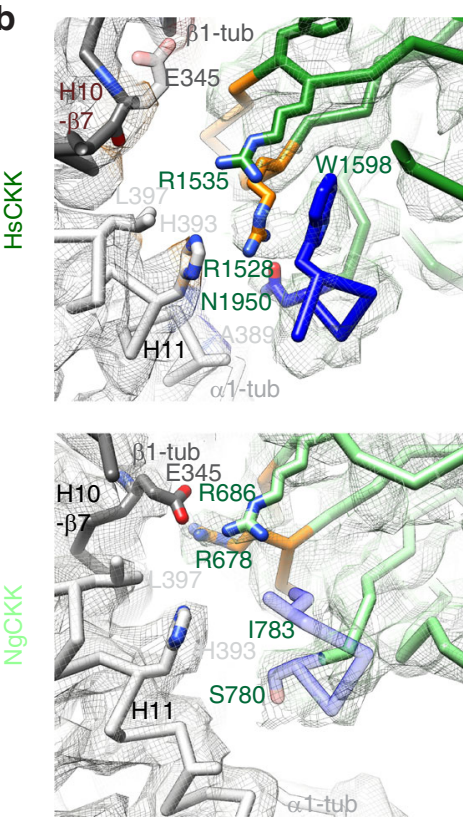

C

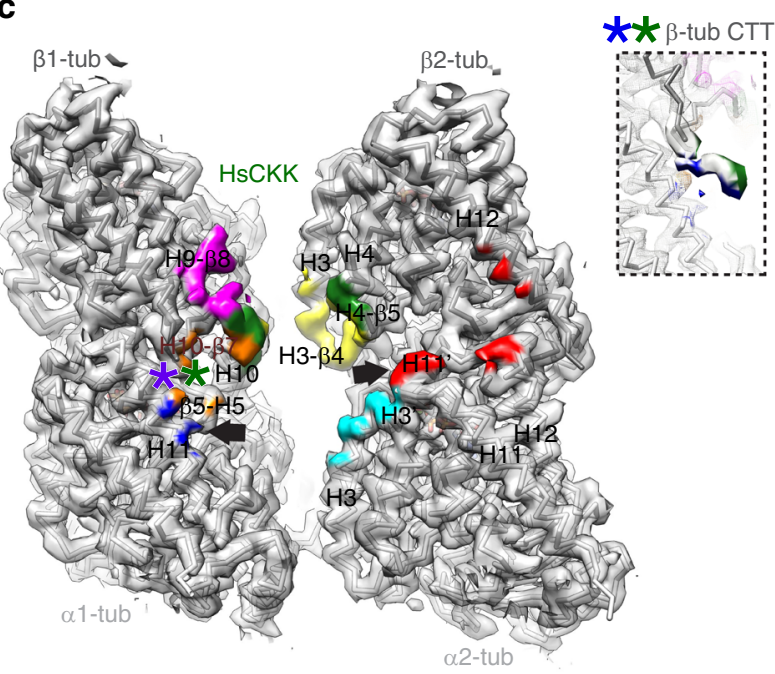

d
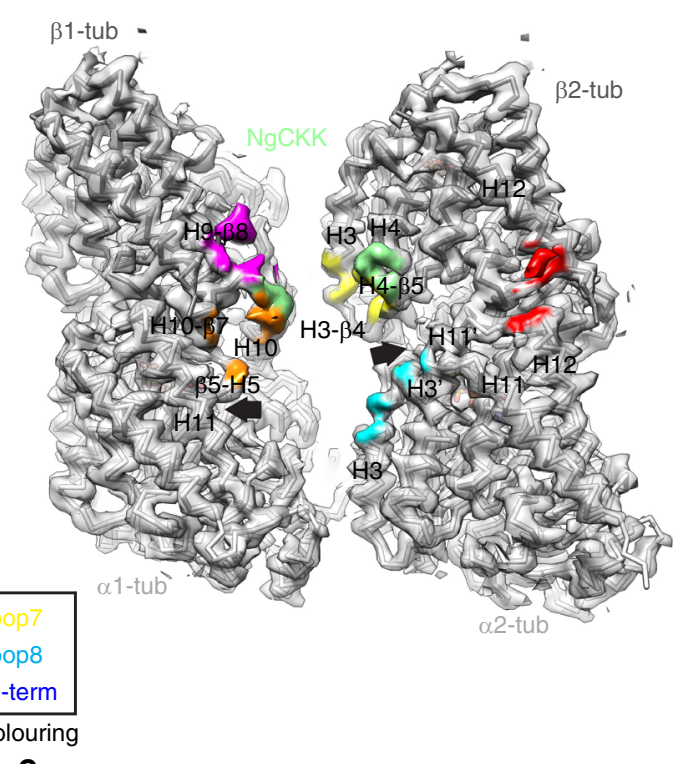

\begin{tabular}{|ll|}
\hline N-term & loop7 \\
loop1 & loop8 \\
loop3 & C-term \\
\hline
\end{tabular}

e

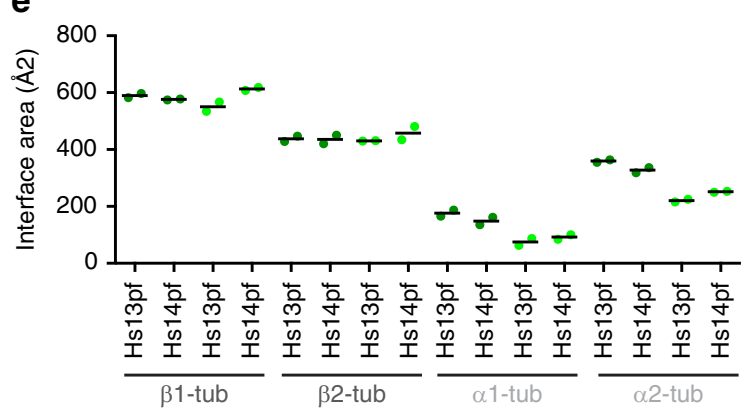

(equivalent to R1535) also interacts with $\beta 1$-tubulin, extending to within hydrogen bonding distance of E345.

Altogether, the subtle differences within the NgCKK sequence, fold and positioning of the domain relative to tubulin result in a different binding footprint on the MT surface compared to HsCKK (Fig. 2c,d). Both the new HsCKK structure and the $\mathrm{NgCKK}$ structure exhibit the previously described larger interface with $\beta$-tubulin compared to a-tubulin (Fig. 2e) proposed to be important for minus end recognition ${ }^{2}$. Intriguingly, however, the differences between $\mathrm{HsCKK}$ and $\mathrm{NgCKK}$ contacts are most striking on $\alpha$-tubulin, with the HsCKK C-terminus/loop3 and loop8/N-terminus forming closer contacts with $\alpha 1$ - and $\alpha 2$ - tubulin, respectively, compared to $\mathrm{NgCKK}$. Indeed, the overall $\mathrm{NgCKK}$ footprint is smaller on both $\alpha 1$ - and a2-tubulin compared to HsCKK (Fig. 2e). These data thereby highlight that the role of contacts with $a$-tubulins in mediating the minus end specificity of $\mathrm{HsCKK}$ is more sophisticated than was previously proposed (see Discussion).

Sensitivity of HsCKK to microtubule lateral curvature. In binding between two tubulin dimers, CKK domains are well placed to sense changes in inter-tubulin lateral curvature, which affects the distance and angle between adjacent protofilaments. 
Fig. 2 MT-bound NgCKK and HsCKK structures and binding footprints are subtly different. a Differences in HsCKK and NgCKK positioning relative to tubulin (used for alignment and shown as white backbone model) viewed from the MT lumen; top, overlay of HsCKK (dark green) and NgCKK (light green) backbones; bottom, backbone RMSD between HsCKK and NgCKK shown on the HsCKK structure, demonstrating their shifted binding position relative to tubulin particularly in tubulin-contacting loop8 and loop3. b Comparison of the contacts formed between HsCKK or NgCKK and $\alpha$-tubulin H11 (light grey backbone) and $\beta$-tubulin's H10- $\beta 7$ loop (dark grey backbone); top, HsCKK contacts with tubulin; bottom, NgCKK contacts with tubulin; cryo-EM density is shown in mesh and the CKK model loops are coloured according to CKK Secondary Structure Element (SSE)/loop colour scheme in the box. c Overview of the HsCKK MT-binding footprint on its intradimer interprotofilament binding site; tubulin density $<5 \AA$ from HsCKK is coloured according to the CKK SSE/ loop colour scheme in $\mathbf{b}$; asterisks refer to the inset, which shows HsCKK interaction with the $\beta$-tubulin CTT; arrows indicate regions where tubulin contacts differ in $\mathrm{HsCKK}$ and $\mathrm{NgCKK}$. d The footprint of $\mathrm{NgCKK}$ on the MT is different compared to HsCKK. Tubulin density $<5 \AA$ from the CKK is coloured according to CKK SSE/loop colour scheme in $\mathbf{b}$. Arrows indicated regions where tubulin contacts differ in $\mathrm{HsCKK}$ and $\mathrm{NgCKK}$. e Calculated interface area in $\AA^{2}$ between $\mathrm{HsCKK}$ or NgCKK and dimer pair subunits, $\alpha 1$-tubulin, $\alpha 2$-tubulin, $\beta 1$-tubulin and $\beta 2$-tubulin, for both 13 - and 14 -protofilament reconstructions, showing smaller $\alpha$-tubulin contacts in NgCKK compared to HsCKK. For each dataset, two measurements (light/dark green points) were made in PISA using the models refined into each independent half-data reconstruction, the mean of which is also shown (black line); source data are provided as a Source Data file

Previously, we proposed that sensitivity to lateral curvature was an important facet of HsCKK binding minus-end specificity. Since MTs with different protofilament numbers exhibit different lateral curvature, comparison of CKK binding in our 13- and 14protofilament reconstructions allowed us to investigate this effect. Superposition of a single tubulin dimer from each of the 13- and 14-protofilament atomic models for $\mathrm{NgCKK}$ and $\mathrm{HsCKK}$ reconstructions shows that the interprotofilament lateral angle in 14- compared to 13-protofilament MTs is $\sim 2^{\circ}$ shallower (Fig. 3). In response, $\mathrm{NgCKK}$ is only slightly altered in its binding site on 14-protofilament MTs (Fig. 3a, b, Supplementary Movie 2), but HsCKK experiences a larger displacement out of the interprotofilament cleft on 14-protofilament MTs (Fig. 3c, d, Supplementary Movie 3). Even though the HsCKK interface with $\alpha$ tubulin remains larger than that of $\mathrm{NgCKK}$ on 14-protofilament MTs (Fig. 2e), the comparison between different MT architectures suggests that in the context of decreased lateral curvature, HsCKK is more prone to being squeezed out of its binding site than $\mathrm{NgCKK}$. This is presumably because it binds deeper between protofilaments compared to NgCKK (Fig. 3).

Since our model of minus-end recognition predicts that lateral flattening of adjacent a-tubulin pairs would favour $\mathrm{HsCKK}$ binding, the outward displacement of HsCKK from its binding site on slightly flatter 14-protofilament MTs initially appears counter-intuitive. In the lattice, however, lateral flattening affects $\alpha$ - and $\beta$-tubulins equally, whereas our model suggested that both (i) lateral flattening in $\alpha$-tubulin towards the MT minus end is particularly important and furthermore that (ii) preferential lateral flattening in adjacent $\beta$-tubulin pairs at MT plus ends would disfavour HsCKK binding. Thus, the potential enhancement of a-tubulin contacts in 14-protofilament MTs is balanced by tightening at the already tight $\beta$-tubulin interface. This emphasises the sensitivity to subtle differences in tubulin conformation encoded by HsCKK that supports its MT minusend binding preference, in particular the importance of conformational asymmetry in $\alpha$ - and $\beta$-tubulins at this site.

CKK protofilament skew correlates with minus end specificity. In addition to comparison of the NgCKK and HsCKK binding sites, the overall architecture of the decorated MTs can be compared to shed further light on HsCKK minus-end preference. We previously described the ability of $\mathrm{HsCKK}$ to induce positive (right-handed) protofilament skew in MTs polymerized from mammalian brain tubulin. Raw images (Fig. 4a) and particle alignment parameters (Fig. 4b) from our new HsCKK-MT dataset support this observation on tsA201 cell-tubulin MTs. 13protofilament MTs usually have unskewed protofilaments, running straight along the MT wall (Fig. 4c), whereas HsCKK binding causes right-handed protofilament skew (Fig. $4 \mathrm{a}-\mathrm{c}$ ).
Furthermore, 14-protofilament MTs usually have negatively skewed protofilaments but HsCKK binding caused these protofilaments to lie parallel to the MT wall with no skew (Fig. 4a, b). In other words, we observed induction of right-handed protofilament skew by $\mathrm{HsCKK}$ in both types of MT architectures. Intriguingly - and in contrast to the $\mathrm{HsCKK}$ - the intrinsic protofilament skew in both 13- and 14-protofilament MTs was unperturbed by NgCKK binding (Fig. $4 \mathrm{a}-\mathrm{c}$ ). This is consistent with the idea that protofilament skew induction correlates with MT minus-end specificity.

Protofilament skew can arise either from a stagger of individual subunits along a protofilament perpendicular to the long axis of the protofilament, or from tilt of the whole-protofilament relative to the MT axis ${ }^{30}$. The higher resolution of our new reconstructions allowed us to probe how protofilament skew is structurally accommodated in HsCKK-bound MTs and thereby shed light on requirements for tubulin plasticity to support HsCKK minus end recognition. To do this, we aligned and compared the HsCKK and NgCKK C1 cryo-EM reconstructions; this is because although these structures have slightly lower resolutions than the symmetrised reconstructions, the fact that they have not been symmetrised means they more closely reflect the overall polymer structure. When protofilaments of NgCKK and HsCKK models fitted into their corresponding $\mathrm{C} 1$ reconstructions are then overlaid, a positive skew of HsCKK protofilaments relative to $\mathrm{NgCKK}$ protofilaments is observed (clockwise rotation viewed from the outer surface the MT, Fig. 4d, left). This skew is reflected in increasing RMSD between the two models along the helical axis (shown in Fig. 4d, right). However, when the models of individual protofilament from $\mathrm{NgCKK}$ and $\mathrm{HsCKK}$ structures are directly aligned, this produces only a small RMSD $(<1 \AA)$ along the whole protofilament (Fig. 4e), i.e. the structures of protofilaments from each reconstruction are essentially the same. This comparison shows that, rather than rearrangements within protofilaments, protofilament skew in HsCKK-bound MTs results from a tilt of whole protofilaments relative to the pseudo-helical axis (Fig. 4f).

Free and MT-bound CKK domains are remarkably rigid. What are the properties of HsCKK compared to $\mathrm{NgCKK}$ that support induction of whole-protofilament skew? To answer this question at atomic resolution we first turned to solid-state NMR (ssNMR). To collect the highest quality ssNMR data, a high affinity HsCKK-MT interaction was required. Our previous work identified an HsCKK mutant, N1492A, that increased the binding affinity for MTs compared to wild-type HsCKK while reducing its selectivity for MT minus ends. Therefore, a range of NMR data were collected using this mutant, and in line with its previously described MT-binding properties, we observed improved 
a

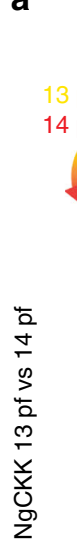

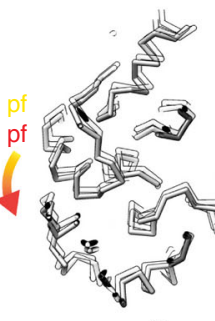
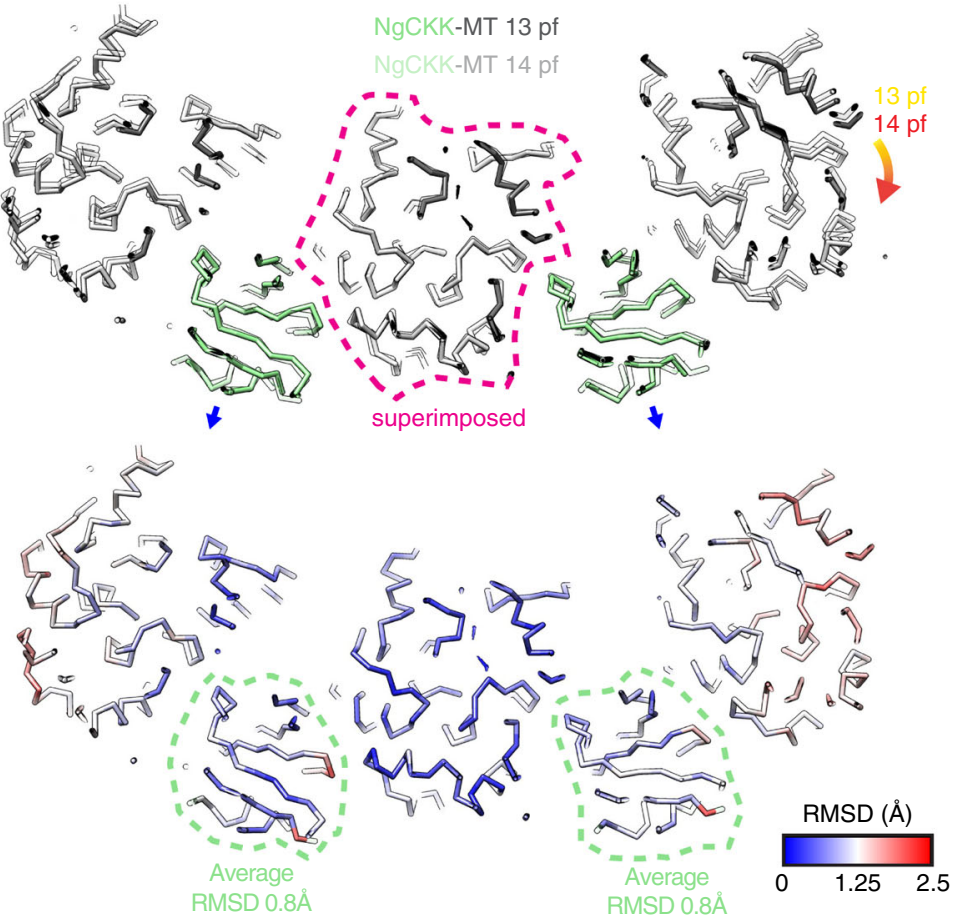

C

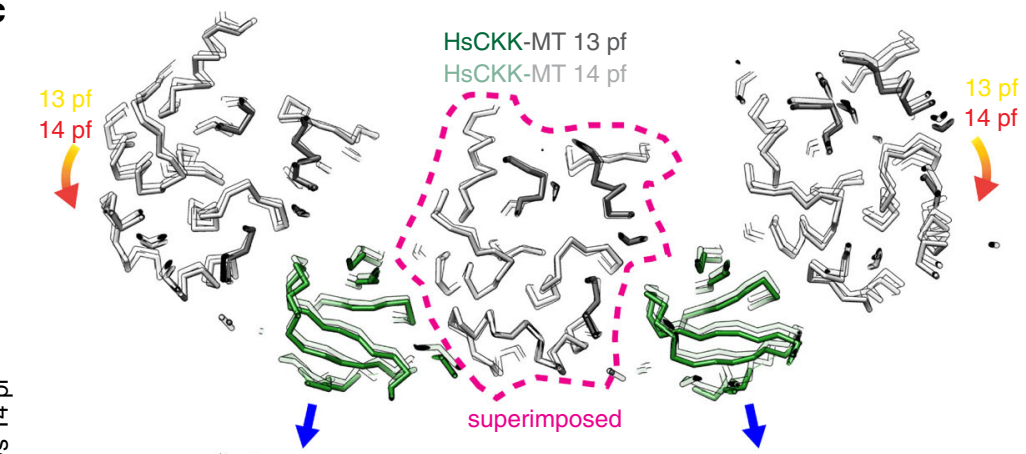

d
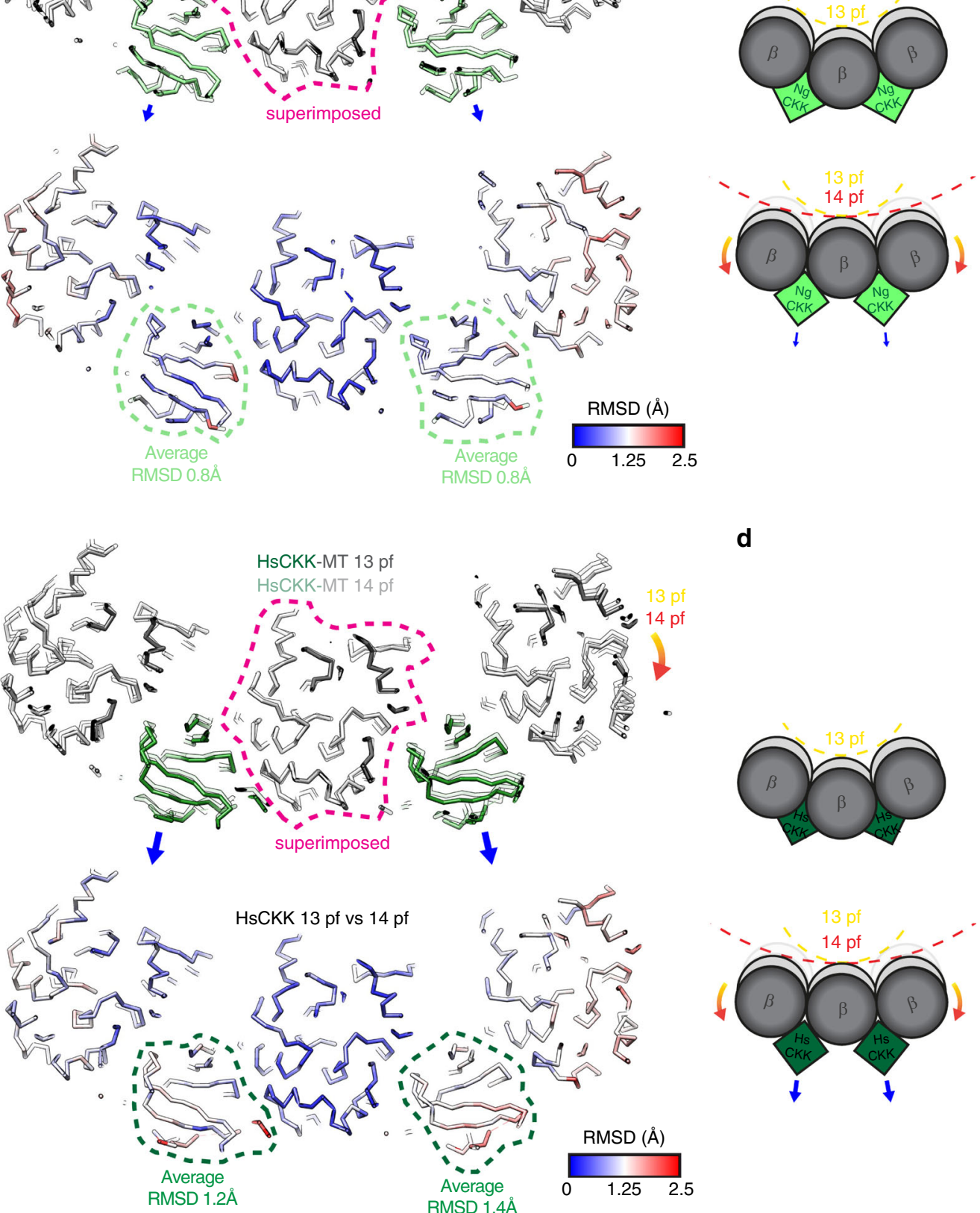

HsCKK 13 pf vs 14 pf
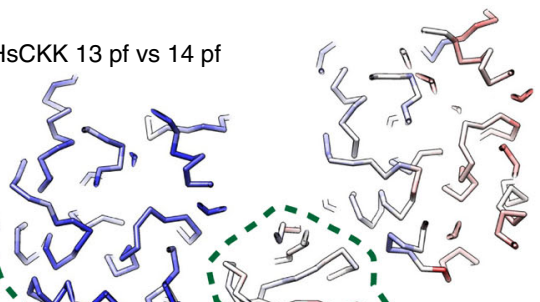

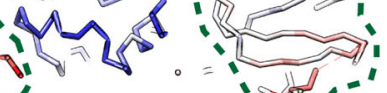

$\operatorname{RMSD}(\AA ̊)$

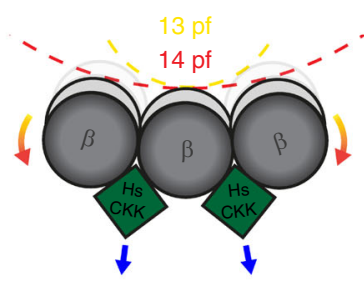

Average

RMSD 1.4Å resolution in our ssNMR spectra compared to the wild-type $\mathrm{CKK}^{2}$ (Supplementary Fig. 6a). Importantly, this gain in spectral resolution allowed us to conduct fast magic angle spinning (MAS), ${ }^{1} \mathrm{H}$ detected ssNMR experiments to further elucidate the CKK conformations in complex with MTs (Supplementary Fig. 6b-d). The chemical-shift perturbations observed upon MTbinding agreed with the previous reported CKK-MT interface while providing a more quantitative and residue-specific description of the behaviour of the domain (Fig. 5a, left). Importantly, we mainly observed chemical-shift perturbations on the amide ${ }^{1} \mathrm{H}$ and ${ }^{15} \mathrm{~N}$ atoms, while no significant chemical-shift perturbations were detected for the $\mathrm{Ca}$ atoms, indicating that the secondary structures of $\mathrm{HsCKK}$ did not alter upon binding to MTs (Supplementary Fig. 6c, d).

In this context, we speculated that despite their overall conserved fold, HsCKK and $\mathrm{NgCKK}$ may have intrinsically different structural properties. To obtain additional insight into the structural properties of these CKK domains, we probed residue-specific dynamics of several free CKK domains using solution-state NMR. Specifically, we conducted Carr-PurcellMeiboom-Gill (CPMG) relaxation dispersion ${ }^{31,32}$ and Chemical Exchange Saturation Transfer (CEST) experiments ${ }^{33}$ to reveal 
Fig. $3 \mathrm{HsCKK}$ is more sensitive to MT lateral curvature than NgCKK. a A transverse slice, viewed from the MT plus end, through 3 protofilaments from the NgCKK-MT 13- and 14- protofilament (pf) models superimposed on the central protofilament. This shows that the adjacent protofilaments adopt a shallower relative angle in 14-protofilament MTs (orange arrows) and reveals the response of $\mathrm{NgCKK}$ bound in the interprotofilament valley to this change in lateral protofilament curvature. Top, view of the superimposed protofilament backbone models from the 13- and 14-protofilament structures superimposed on the central protofilament (dotted pink outline); small blue arrows indicate the relatively small displacement of NgCKK from its binding site on 14-protofilament MTs. Bottom, backbone RMSD between the 13- and 14-protofilament models shown on the 13-protofilament model; $\mathbf{b}$ schematic depicting the effect of MT protofilament architecture on NgCKK binding. $\mathbf{c}$ A transverse slice, viewed from the MT plus end, through three protofilaments from the HsCKK-MT 13- and 14- protofilament models superimposed on the central protofilament. This again shows how the adjacent protofilaments adopt a shallower relatively angle in 14-protofilament MTs (orange arrows) and reveals the response of bound HsCKK to this change in lateral protofilament curvature. Top, view of the superimposed protofilament backbone models from the 13- and 14-protofilament structures superimposed on the central protofilament (dotted pink outline); larger blue arrows indicate the larger displacement of HsCKK from its binding site on 14-protofilament MTs. Bottom, backbone RMSD between the 13- and 14-protofilament models shown on the 13-protofilament model; $\mathbf{d}$ schematic depicting the effect of MT protofilament architecture on $\mathrm{HsCKK}$ binding

possible millisecond time-scale conformational exchange processes of CKK domains. The CPMG profiles of HsCKK_N1492A, HsCKK, the CKK domain from mouse CAMSAP3 and NgCKK all showed no exchange in these CPMG time ranges $(50 \mathrm{~Hz}$ $\sim 1.5 \mathrm{kHz}$ ) (Fig. 5a, right and Supplementary Fig. 6e). This is particularly noteworthy in $\mathrm{NgCKK}$ given the large, flexible loop4 insertion compared to HsCKK (Supplementary Fig. 2a). From our data, we can conclude that motions of loop 4 (which most likely occur on the nanosecond scale) do not influence the millisecond time-scale motional profile of the rest of the protein backbone that is probed in our CPMG and CEST experiments.

Similarly, the results of additional CEST experiments using HsCKK_N1492A speak against slow milli-second time-scale motion in free CKK domains, for example, in residues T1553 and S1585, which exhibited significant chemical-shift changes upon complex formation (Supplementary Fig. 6f). Similar results were obtained for CAMSAP3 CKK (Supplementary Fig. 6g) and NgCKK (Supplementary Fig. 6h). Taken together, our NMR experiments suggest that the $3 \mathrm{D}$ structures of CKK domainswhether they recognize minus-ends or not-are remarkably rigid and, in contrast to many other MAPs, do not undergo structural changes upon MT binding.

HsCKK remodels its binding site causing MT remodelling. We then wanted to know how protofilament skew was brought about by HsCKK binding and accommodated by the MT lattice. The geometric constraints that define MT architecture while maintaining longitudinal and lateral tubulin contacts are captured in the lattice accommodation model ${ }^{34,35}$, and we investigated in turn the set of interconnected structural parameters this model describes.

The helical rise and monomer repeat distances were not significantly different in MTs (13- or 14-protofilament) bound by HsCKK or NgCKK (Supplementary Table 2). However, protofilaments in HsCKK-MTs are closer together (Fig. 5b), giving these MTs a $\sim 4 \AA$ smaller diameter than NgCKK MTs with the same protofilament number. The diameter shrinkage occurs because the centres of mass of all neighbouring B-lattice dimers in a single 3-start helical turn are closer by around $0.4 \AA$ in HsCKKcompared to NgCKK-MTs (Fig. 5c); such a difference is not observed in the equivalent comparison between half-maps within each dataset (Supplementary Fig. 7a). This inward protofilament positioning is not symmetrical around the MT, with a range of 0.4-1.5 $\AA$ relative shifts observed in both 13 - and 14protofilament architectures (Fig. 5b), and with the biggest deviations seen at and opposite of the seam. Again, such a difference is not observed in the equivalent comparison between half-maps within each dataset (Supplementary Fig. 7b). The small compression between lateral neighbouring tubulin dimers in the lattice can be observed by superimposing the ones from the atomic models of NgCKK-MT and HsCKK-MT (Fig. 5d, top). This analysis also reveals a longitudinal displacement of adjacent tubulin dimers in the HsCKK model relative to the $\mathrm{NgCKK}$ model; this is indicative of shearing of adjacent protofilaments as they skew. There are, however, no detectable differences in interprotofilament lateral contacts (small RMSDs, Fig. 5d, bottom; Supplementary Fig. 7c, d). Rather, small adjustments across the outer tubulin surface-where HsCKK binds-flexibly accommodate shifts in the MT architecture due to HsCKK binding (Fig. 5d, bottom, larger RMSDs in the tubulin on the left). In summary, relative to $\mathrm{NgCKK}$, HsCKK induces small conformational changes in tubulin at its binding site which, in the context of whole MTs, induces protofilament tilt, shear, lateral compression and a reduction in MT diameter (Fig. 5e).

\section{Discussion}

To shed light on the MT minus-end binding preference of CAMSAPs, we have structurally compared a CKK domain that does not bind MT minus ends-NgCKK-with the CKK domain from human CAMSAP1 (HsCKK), which mediates CAMSAP1's MT minus-end binding preference. Little is known about the native MT ultrastructure of Naegleria, so it is possible that NgCKK could recognise MT minus ends on Naegleria $\mathrm{MTs}^{36}$. However, for the purposes of our current study, NgCKK has proven an invaluable tool for evaluating MT minus end binding mechanisms on mammalian MTs. To allow a near-atomic resolution investigation of the subtle mechanism(s) at work, we studied NgCKK and HsCKK MT lattice binding in the context of different MT architectures and used these structures to explain the differences in their MT minus-end recognition properties (Fig. 6).

In this study, we found that $\mathrm{NgCKK}$ and HsCKK share the same protein fold, they bind at the same intradimer interprotofilament site and, as revealed using a combination of NMR methodologies, share the same intrinsic structural rigidity. This shows that the presence/absence of MT minus-end recognition is not due to large conformational changes in the domain or significant alterations in the CKK binding site. Although the distinct MT interactions by the flexible regions adjacent to the CKK core have a large effect on MT affinity (Supplementary Fig. 4), the biggest difference between these two domains is that HsCKK forms a more extended interface with the $\alpha$-tubulin pair at its binding site than $\mathrm{NgCKK}$ and sits deeper within the interprotofilament groove. We found that HsCKK is more displaced from its binding site on 14- compared to 13-protofilament MTs, squeezed outwards by the flatter lateral curvature of adjacent protofilaments in the higher protofilament number MT architecture. In addition, HsCKK binding induces positive protofilament skew in both 13- and 14-protofilament MTs while $\mathrm{NgCKK}$ does not; our reconstructions show that, in inducing 
a

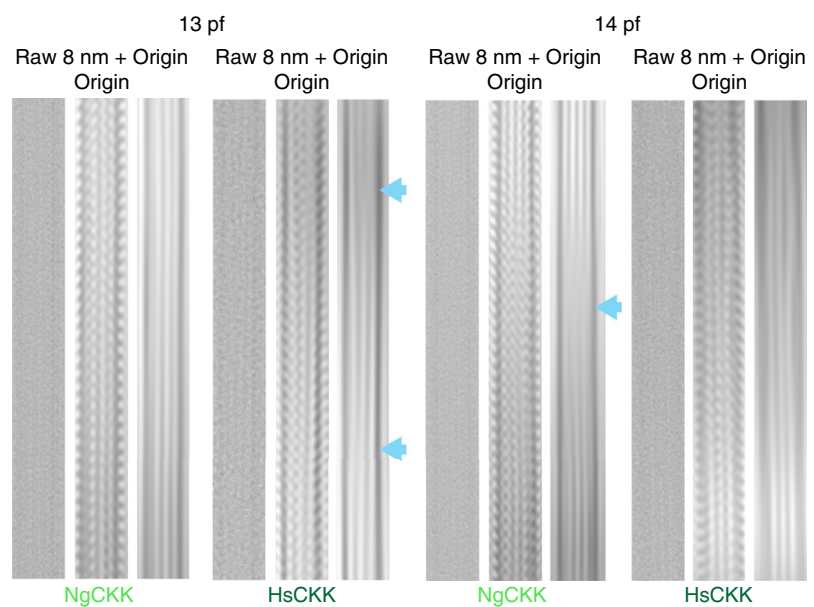

b

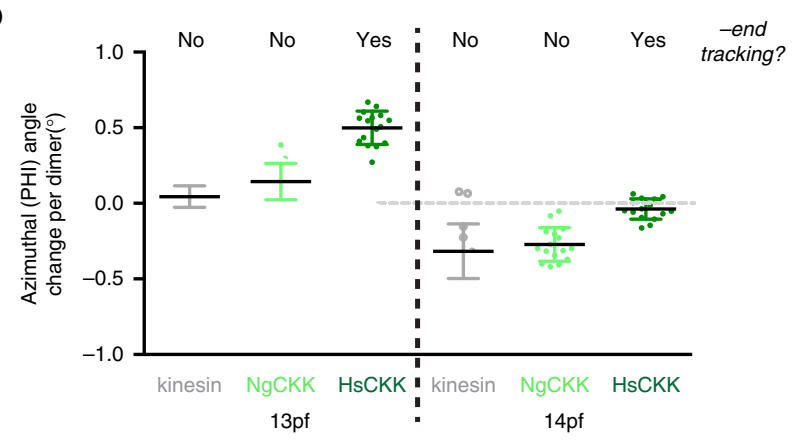

d

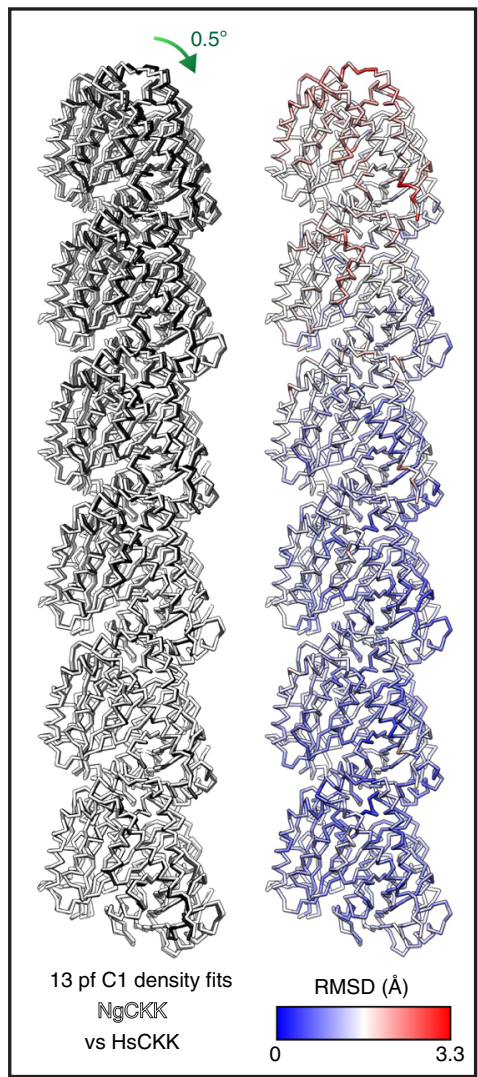

e

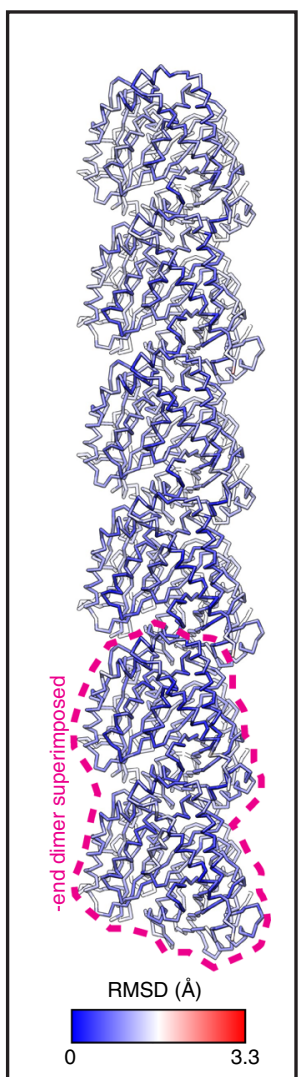

C

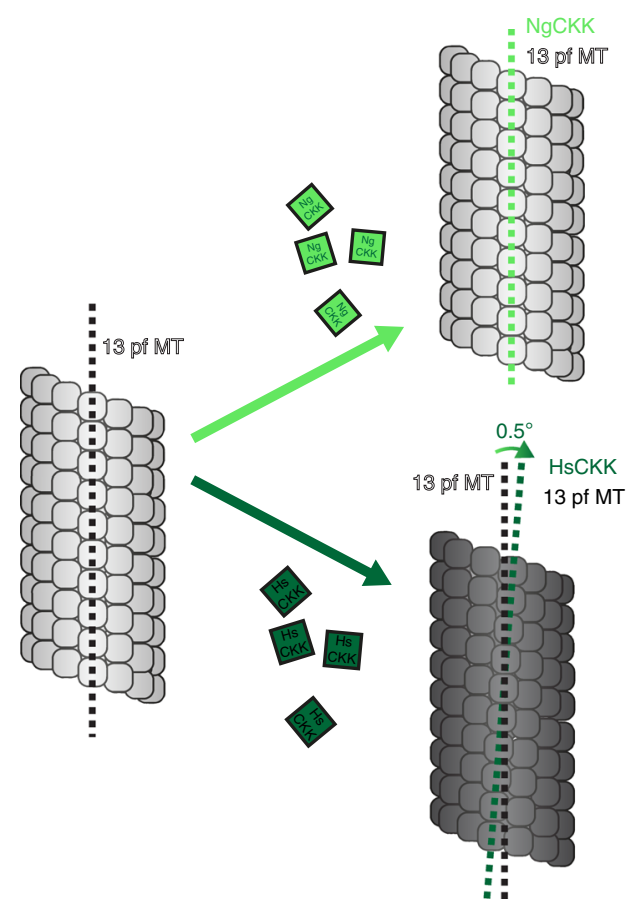

f

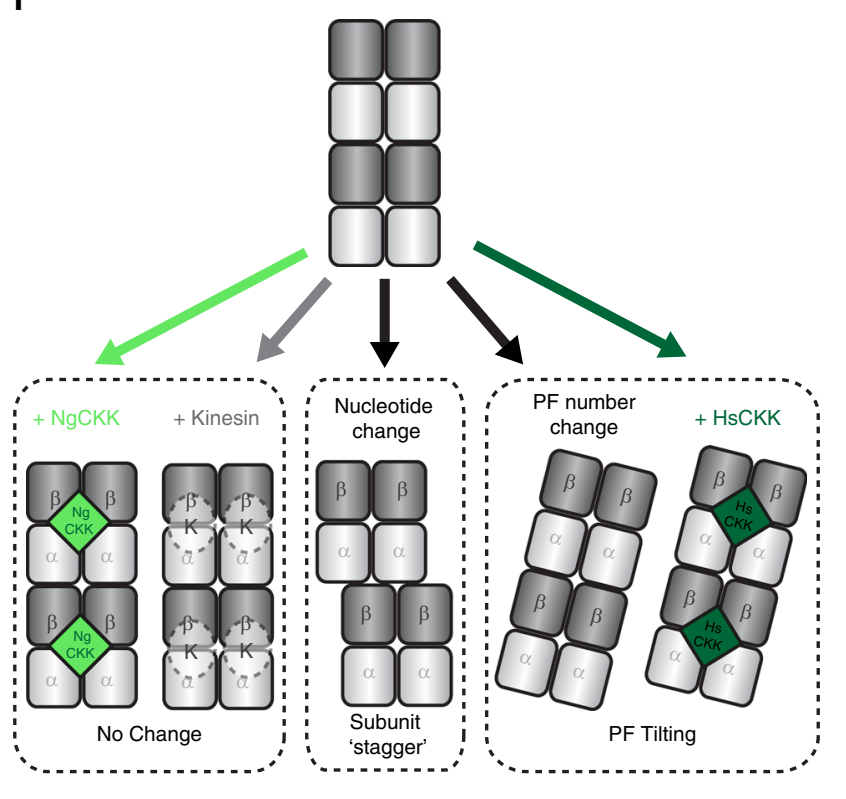

protofilament skew, HsCKK brings the tubulin dimers to which it binds closer together. While neither intra- nor interdimer longitudinal interfaces are perturbed in HsCKK-bound MTs, their global lattice architecture alters to accommodate the local remodelling at the HsCKK binding site: compared to NgCKKMTs, the diameter of HsCKK-MTs are smaller and adjacent protofilaments exhibit compression, shear and skew while conserving the B-lattice MT architecture with a single seam predicted by the lattice accommodation model.

The first important aspect of the MT minus-end recognition mechanism by HsCKK revealed by our current data is that the CKK domain itself does not flexibly respond to different tubulin 
Fig. $4 \mathrm{HsCKK}$, but not NgCKK, induces tilting of whole protofilaments. a Raw and Fourier filtered images of 13- and 14- protofilament MTs decorated by $\mathrm{NgCKK}$ and HsCKK. In each set of three panels: left, raw image; centre, filtered image to include data at, and adjacent to, the origin and the $1 / 8 \mathrm{~nm}$ layer line; right, filtered image to include data at, and adjacent to, the origin highlights the MT moiré pattern; blue arrows indicate variations in the moiré pattern that arise from protofilament skew. b Protofilament skew for a 16 MT subset from each dataset plotted as the average rotation angle around the MT axis (PHI) change per dimer moving axially towards the MT plus-end. HsCKK-decorated MTs compared to kinesin decorated paclitaxel-stabilized MTs (13protofilament kinesin-3 data from 40 ; 14-protofilament kinesin-1 data from ${ }^{40}$ ). All data points are plotted and bars represents mean \pm SD. HsCKK 13 protofilament (tSA201 tubulin) vs kinesin-3 13-protofilament, $p<0.0001, \mathrm{NgCKK} 13$-protofilament vs HsCKK 13-protofilament, $p<0.0001, \mathrm{NgCKK} 13-$ protofilament vs kinesin-3 13-protofilament, not significant ( $p=0.164)$, HsCKK 14-protofilament (tSA201 tubulin) vs kinesin-1 14-protofilament, $p<0.001$, NgCKK 14-protofilament vs HsCKK 14-protofilament, $p<0.0001$, NgCKK 14-protofilament vs kinesin-1 14-protofilament, not significant ( $p=0.889$ ), oneway ANOVA with Tukey's multiple comparisons test; source data are provided as a Source Data file. c Schematic of influence of NgCKK/HsCKK on protofilament skew; d left, MT protofilaments fitted into aligned HsCKK and NgCKK C1 reconstructions were overlaid; the bottom dimer corresponds to the point at which the density was aligned; divergence between NgCKK and HsCKK models increases from this point; right, RMSD of backbone positions in panel (i) depicted on a NgCKK protofilament; e RMSDs between NgCKK and HsCKK protofilaments calculated when the bottom dimers in the models themselves are directly aligned, where RMSD does not decrease significantly with distance from the superimposed dimer; $\mathbf{f}$ mechanisms of protofilament skew induction: left, no skew change; middle, protofilament skew arising from interdimer subunit stagger, e.g. from tubulin GTPase; right, protofilament skew arising from whole-protofilament tilting e.g. due to changes in protofilament number or HsCKK binding

conformations. Rather, its rigidity is consistent with its sensitivity to, and affinity for, the conformation(s) of polymerized tubulin it encounters. Second, we confirmed that the ability to induce significant protofilament skew in fully decorated MTs correlates with MT minus-end recognition activity. This was previously observed in the HsCKK-N1492A mutant but is now confirmed in the comparison of HsCKK with NgCKK. Third, we previously speculated that skew induction reflects the non-optimal geometry for HsCKK binding of tubulin dimers within the MT lattice compared to minus ends. Our new reconstructions show that this is indeed the case, and that skew arises in response to HsCKK forcing the two tubulin dimers it contacts closer together. Consistent with this idea, the CKK binding site on neighbouring tubulin dimers are predicted to be laterally closer in the transition zone to gently curved tubulin sheets near MT minus ends which HsCKK prefers. Fourth, a key prediction of our previous model is that end specificity by $\mathrm{HsCKK}$ is mediated by the asymmetric curvature of tubulin at the minus end, with the $\alpha$-tubulins less laterally curved relative to the $\beta$-tubulins. Conversely, at plus ends - with the $\beta$-tubulins less laterally curved-HsCKK binding is inhibited. We previously hypothesised that looser contacts between HsCKK and $\alpha$-tubulins compared to $\beta$-tubulins are an important aspect of MT minus end recognition. In fact, this difference in contact area between tubulins is seen in both HsCKK and NgCKK structures (Fig. 2e). Thus, while this aspect of the model provides a logical explanation for the reduced affinity of HsCKK for MT plus ends ${ }^{2}$ (Fig. 6), we now show that, by itself, tighter contact between $\beta$ - compared to $\alpha$-tubulin is insufficient to explain HsCKK minus end preference. However, HsCKK forms different and more extensive contacts with $\alpha$ tubulins compared to $\mathrm{NgCKK}$; the looser interactions between $\mathrm{NgCKK}$ and the $\alpha$-tubulins suggest that sensitivity to tubulin configuration is thereby absent (Fig. 6) and explains why this protein does not specifically bind MT minus ends. Therefore, our new data show that, while looser than the contacts with $\beta$-tubulin, the HsCKK contacts with $\alpha$-tubulins are critical in enabling it to recognise and bind to the relatively flattened configuration of $\alpha$ tubulins at minus-ends.

Structural studies of MT-bound MAPs typically reveal conformational changes in the MAP on interaction with the MT lattice. In the most extreme cases, unstructured proteins such as members of the tau/MAP2 family and the mitotic regulatory protein TPX2, become at least partially ordered when in contact with $\mathrm{MTs}^{37,38}$. A recent study of the plant MAP Companion of Cellulose synthase 1 also showed similar behaviour in its disordered N-terminus on binding $\mathrm{MTs}^{39}$. Folded MT binding domains in a number of MAPs-for example, kinesin motor domains ${ }^{40,41}, \mathrm{CH}$ domains in EB proteins ${ }^{42}$, the p150glued CAPGly domain ${ }^{43}$-often undergo some rearrangements and/or ordering of otherwise disordered loop regions on formation of the MT-bound complex. Although the termini adjacent to the CKK become ordered on MT binding, we show that the core of HsCKK, which is essential for minus end recognition, is sufficiently rigid that it does not undergo conformational changes on MT interaction, but rather the MT lattice is remodelled in response to HsCKK binding. In the case of $\mathrm{HsCKK}$, this is because the main MT lattice is not the preferred binding substrate for CKK. However, this behaviour-in which a structurally invariant MAP is exquisitely sensitive to the precise conformation of the underlying tubulin-is likely to be shared by other proteins.

The availability of increasing numbers of MT structures bound by a range of ligands has also emphasised that, far from being an inflexible, structurally invariant cylinder, the MT lattice supports surprising structural plasticity. Lattice compaction at the interdimer interprotofilament tubulin contacts in response to the tubulin GTPase is well documented in MTs polymerized from mammalian tubulin $30,42,44-46$. End Binding (EB) proteins bind at the corner of four tubulin dimers adjacent to the tubulin GTPase site $^{47}$ and their preference for the sleeve of GDP.Pi compacted tubulins that dynamically evolves as MTs grow mediates their tiptracking activity ${ }^{42,47-49}$. EB binding itself induces a small lefthanded protofilament skew by introducing a slight interdimer stagger along the protofilament, the mechanistic significance of which is not yet understood. CKKs bind MTs $4 \mathrm{~nm}$ away from the EB binding site and are insensitive to nucleotide-dependent conformational changes in the lattice ${ }^{2}$. We show here that HsCKK binding induces right-handed protofilament skew via tilt and shear of entire protofilaments, a completely different mechanism than seen for EBs. Thus, our characterization of the HsCKK-MT interaction also highlights the extent of structural plasticity that can be accommodated in the MT lattice. Small conformational effects induced by one MAP could have substantial consequences for binding of other MT-binding factors. We previously demonstrated that CKK binding at MT ends can sterically compete with kinesin-13 at MT minus ends ${ }^{2}$, thereby protecting them from depolymerisation ${ }^{21-23}$. Our current work also suggests that, beyond direct steric competition, different MAPs may exert allosteric control over each other's MT binding by modifying the conformation of the MT lattice ${ }^{30}$. This was also suggested by a recent study of axonemal dynein ${ }^{50}$. Taken together, our data support the idea that MTs can act as allosteric signalling platforms, in which the precise configuration of polymerized tubulins are influenced by their dynamic state and binding partners ${ }^{6,51}$. In the case of CAMSAPs/Patronins, 
a
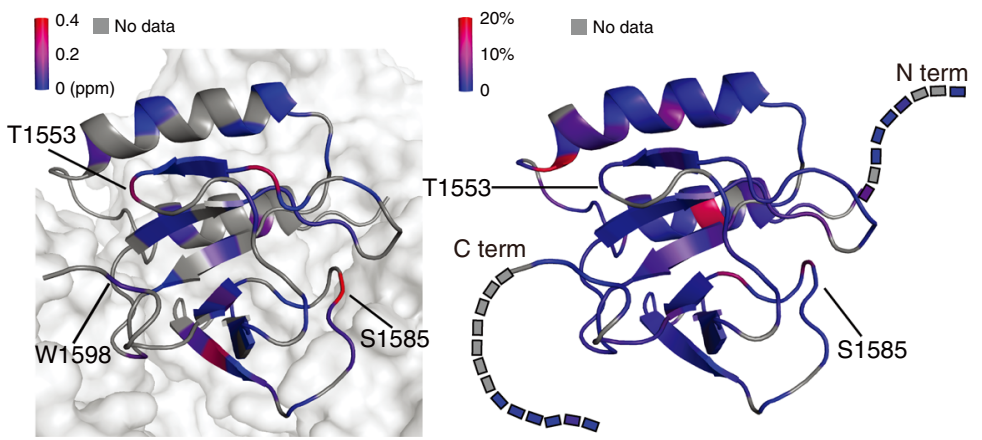

b

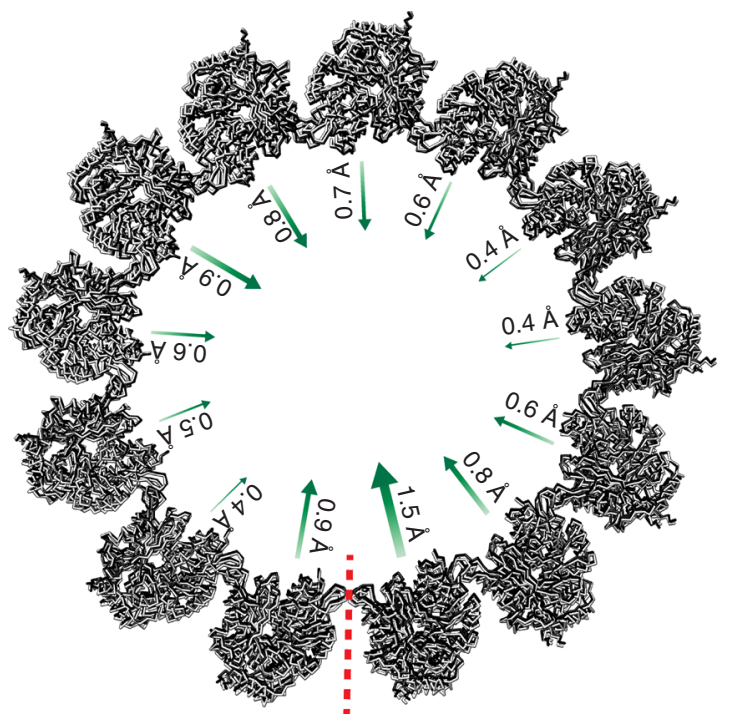

d
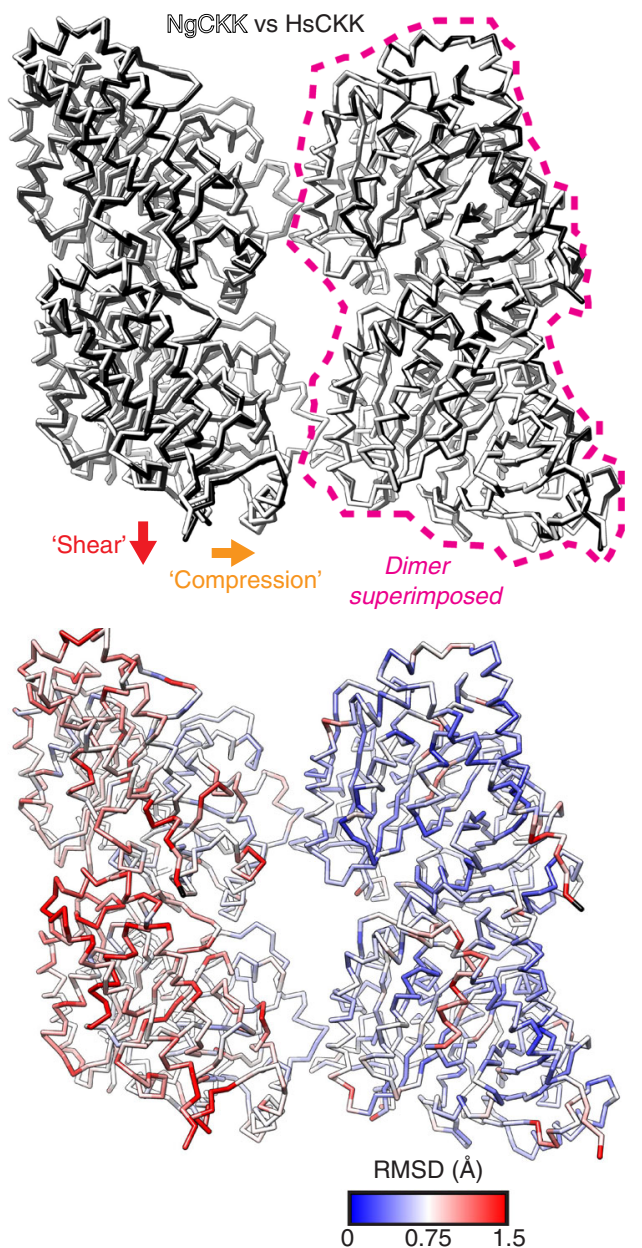

C

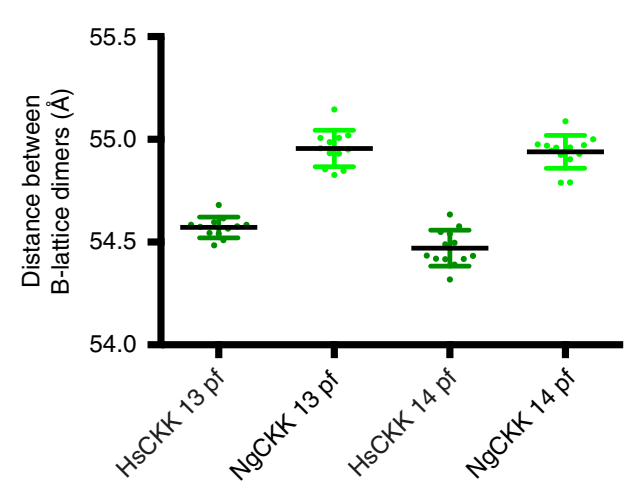

e
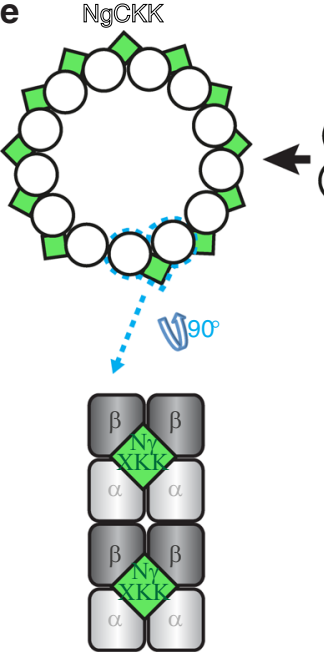

No-end preference
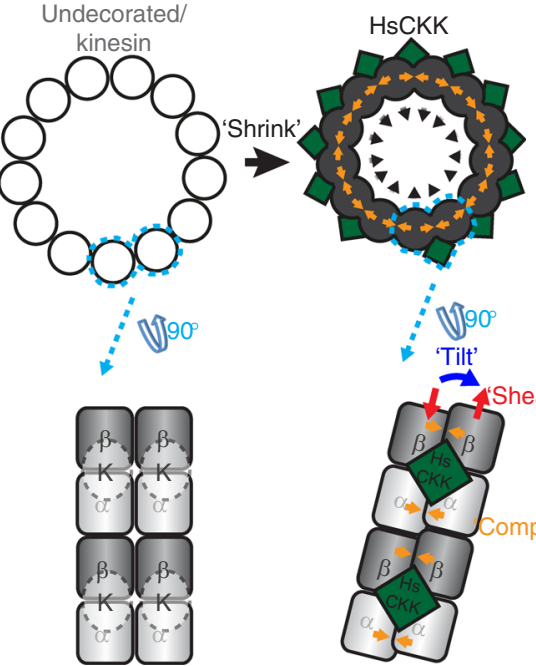

No-end preference
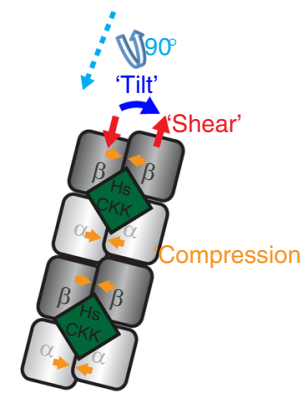

-end

preference sensitivity to structural variations in tubulin is essential for MT minus end recognition. These insights will inform future mechanistic investigations of conformational signalling arising from the MT cytoskeleton.

\section{Methods}

Protein expression and purification for TIRF microscopy. Strep-GFP-tagged human CAMSAP1 CKK (residues 1474-C), Naegleria gruberi CKK (residues 612-
C, reference sequence XM_002675733.1) and chimeric or truncated CKK constructs were prepared as described previously ${ }^{2}$. Briefly, proteins were expressed in HEK293T cells using a modified pTT5 expression vector (Addgene no. 44006), purified using StrepTactin beads (GE). After incubation with the cell lysate, beads were washed five times with high salt wash buffer (50 mM HEPES, $1.5 \mathrm{M} \mathrm{NaCl}$ and $0.01 \%$ Triton X-100), and proteins were eluted in elution buffer (50 mM HEPES, $150 \mathrm{mM} \mathrm{NaCl}, 1 \mathrm{mM} \mathrm{MgCl} 2,1 \mathrm{mM}$ EGTA, $1 \mathrm{mM}$ dithiothreitol (DTT), $2.5 \mathrm{mM}$ dDesthiobiotin and $0.05 \%$ Triton X-100, pH 7.4). Purified proteins were snap-frozen and stored at $-80^{\circ} \mathrm{C}$. Truncated constructs were as follows: $\operatorname{NgCKK} \Delta \mathrm{N}$ 
Fig. $5 \mathrm{HsCKK}$ domain rigidity mediates local remodelling of its MT-binding site. a Left, chemical-shift perturbations, coloured by differences in ppm, arising from HsCKK_N1492A binding to MTs mapped on the structure of CKK-MT complex (PDB: 5M5C); the CKK domain is depicted in a ribbon representation while the MT surface is shown as a space-filling model; Right, changes of transverse relaxation rates obtained from solution-state NMR CPMG experiments plotted on the 3D structure of the free HsCKK domain; source data are provided as a Source Data file. $\mathbf{b}$ HsCKK MT binding is accompanied by contraction of the MT diameter; a single turn of models docked within the aligned 13-protofilament HsCKK and NgCKK C1 reconstructions are shown viewed from the minus end; arrows indicate the irregular shift of individual protofilaments. c Contraction of MT diameter is caused by shrinkage of the distance between adjacent dimers; the distance between the centre of masses of each pair of adjacent B-lattice dimers was measured in 13-and 14-protofilament C1 HsCKK and $\mathrm{NgCKK}$ models; all data points are plotted and bars represents mean $\pm \mathrm{SD}$; differences between $\mathrm{HsCKK}$ and $\mathrm{NgCKK}$ models are statistically significant $(p<0.0001$, t-test); source data are provided as a Source Data file. $\mathbf{d}$ Top, alignment of a single dimer from the NgCKK-tubulin and HsCKK-tubulin C1 models shows HsCKK induces compression and shear between the dimers at its binding site; bottom, RMSD of backbone positions in top panel. e Schematic summarising modifications imposed by HsCKK but not kinesin or NgCKK binding on MT architecture; modifications are exaggerated for clarity
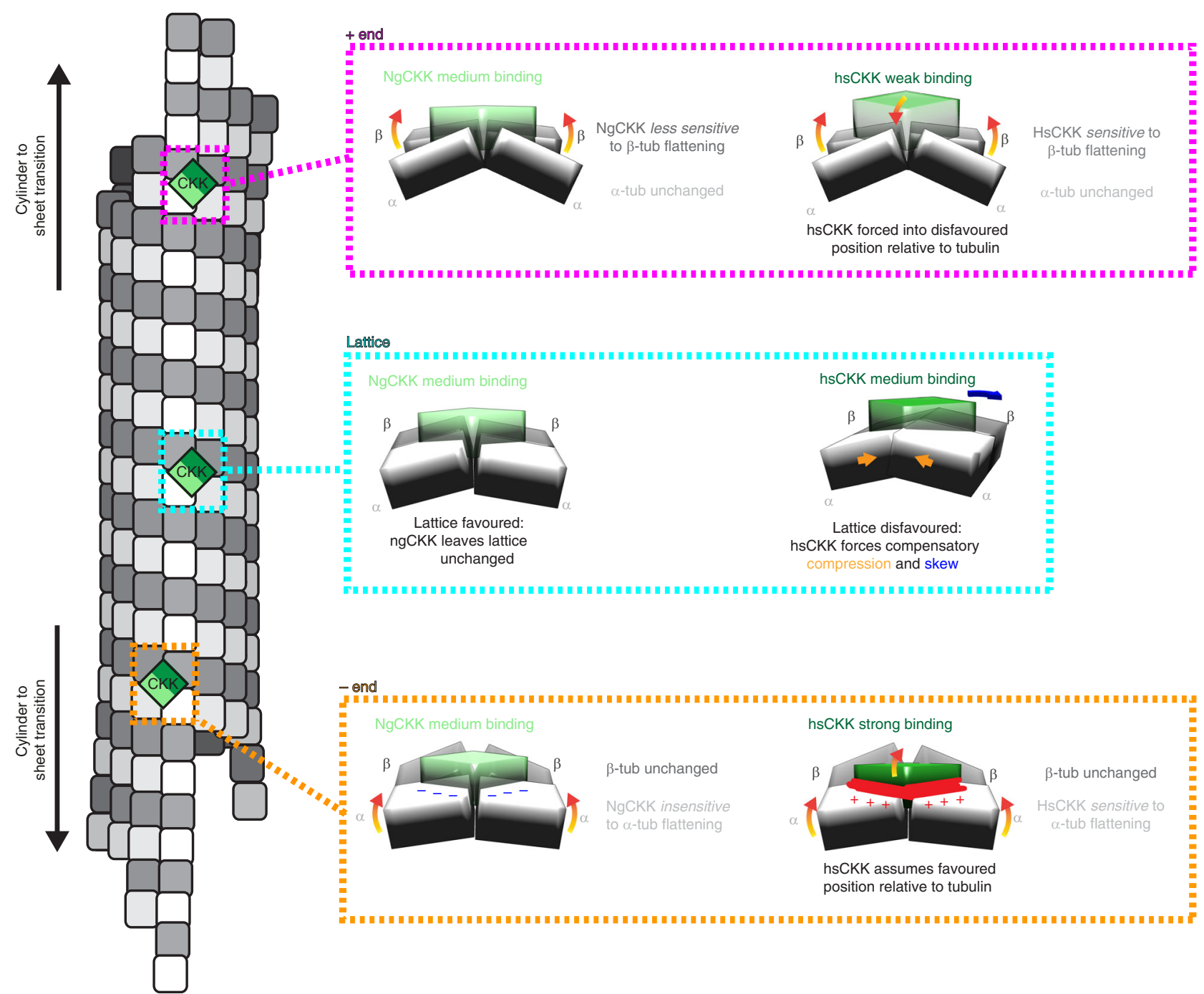

Fig. $6 \mathrm{NgCKK}$ and HsCKK comparison illuminates the CAMSAP MT minus end recognition model. The schematic of a stable/growing 13-protofilament MT on the left shows different zones within the polymer in which the tubulins adopt subtly different conformations. Towards MT ends, there is a region of transition from the cylindrical lattice to curved sheet-like regions in which interprotofilament connections are maintained but which exhibit decreasing lateral curvature and increasing longitudinal curvature away from the MT lattice. Beyond this transition zone, protofilaments gradually terminate and separate at each MT end. On the right, the interaction of $\mathrm{NgCKK}$ and $\mathrm{HsCKK}$ with the unique tubulin dimer pair conformation in each zone is compared. On the MT lattice (middle, cyan boxed zone), as visualised in our cryo-EM reconstructions, NgCKK and HsCKK bind at the same site, but HsCKK remodels its binding site by compressing the tubulin dimer pairs, inducing protofilament skew. At the plus end lattice-end transition (top, pink boxed zone), we hypothesise that $\mathrm{NgCKK}$ is insensitive to the plus end specific tubulin sheet curvature found here, whereas HsCKK binding is actively disfavoured because of the enhanced lateral flattening in the $\beta$-tubulin pair that is specific to the MT plus end. At the minus end lattice-end transition (bottom, orange boxed zone), we again hypothesise that $\mathrm{NgCKK}$ is insensitive to the minus end specific sheet curvature. In contrast, HsCKK binding is favoured because, in the context of its particular tubulin interaction, the specific asymmetrically curved configuration of the tubulin pair-in particular the enhanced lateral flattening in the $\alpha$-tubulin pair-is the preferred binding site conformation for $\mathrm{HsCKK}$ 
corresponded to N. gruberi CKK core and C-terminal extension (633-788), NgCKK $\Delta \mathrm{C}$ to the $N$. gruberi $\mathrm{N}$-terminal extension and CKK core (612-784) and NgCKK $\Delta \mathrm{N}+\mathrm{C}$ consisted of the $N$. gruberi CKK core (633-784). Chimeric constructs were as follows: the NgCKK Swap C construct consisted of N. gruberi Nterminal extension and CKK core (612-784) followed by the HsCKK C-terminal extension (1600-1613), the NgCKK Swap N construct consisted of N. gruberi CKK core and C-terminal extension (633-788) preceded by the HsCKK N-terminal extension (1463-1483). The NgCKK Swap N + C construct consisted of N. gruberi CKK core (633-784) preceded by the HsCKK N-terminal extension (1463-1483) and followed by the HsCKK C-terminal extension (1600-1613). Primer sequences used to prepare these constructs using Gibson assembly are provided in Supplementary Table 3.

TIRF microscopy analysis of CKK binding to dynamic MTs. TIRF microscopy was performed on an inverted research microscope Nikon Eclipse Ti-E (Nikon) with the perfect focus system (PFS) (Nikon), equipped with a Nikon CFI Apo TIRF $\times 100$ 1.49-NA oil objective (Nikon) and a Photometrics Evolve 512 EMCCD (Roper Scientific) camera or a Photometrics Prime BSI camera, and controlled with MetaMorph 7.7 software (Molecular Devices, CA). Images were projected onto the chip of an Evolve 512 camera with an intermediate $\times 2.5$ lens (Nikon $\mathrm{C}$ mount adaptor $\times 2.5$ ). To keep in vitro samples at $30^{\circ} \mathrm{C}$, we used an INUBG2E-ZILCS (Tokai Hit) stage-top incubator.

For excitation, we used $491 \mathrm{~nm} / 100 \mathrm{~mW}$ Stradus (Vortran) and $561 \mathrm{~nm} / 100$ $\mathrm{mW}$ Jive (Cobolt) lasers. For simultaneous imaging of green and red fluorescence, we used a triple-band TIRF polychroic filter (ZT405/488/561rpc, Chroma) and triple-band laser emission filter (ZET405/488/561 m, Chroma), mounted in the metal cube (91032, Chroma) together with an Optosplit III beam splitter (Cairn Research) equipped with a double-emission-filter cube configured with ET525/50 $\mathrm{m}$, ET630/75 $\mathrm{m}$ and T585LPXR (Chroma) filters.

Doubly cycled GMPCPP-stabilized MT seeds were prepared as described before ${ }^{52}$, by incubating the tubulin mix containing $70 \%$ unlabeled porcine brain tubulin (Cytoskeleton), 18\% biotin-tubulin (Cytoskeleton) and 12\% rhodaminetubulin (Cytoskeleton) at a total final tubulin concentration of $20 \mu \mathrm{M}$ with $1 \mathrm{mM}$ GMPCPP (Jena Biosciences) at $37^{\circ} \mathrm{C}$ for $30 \mathrm{~min}$. MTs were pelleted by centrifugation in an Airfuge for 5 minutes at $119,000 \times g$ and then depolymerized on ice for 20 minutes. This was followed by a second round of polymerization at 37 ${ }^{\circ} \mathrm{C}$ with $1 \mathrm{mM} \mathrm{GMPCPP}$. MT seeds were then pelleted as above and diluted 10 -fold in MRB80 buffer ( $80 \mathrm{mM}$ PIPES, pH 6.8, supplemented with $4 \mathrm{mM} \mathrm{MgCl}_{2}$ and 1 mM EGTA) containing $10 \%$ glycerol, snap frozen in liquid nitrogen and stored at $-80^{\circ} \mathrm{C}$.

The in vitro reconstitution assays with dynamic MTs were performed under the same conditions as described previously ${ }^{2}$. Briefly, after coverslips were functionalized by sequential incubation with $0.2 \mathrm{mg} / \mathrm{ml}$ PLL-PEG-biotin (Susos) and $1 \mathrm{mg} / \mathrm{ml}$ neutravidin (Invitrogen) in MRB80 buffer, GMPCPP-stabilized microtubule seeds were attached to the coverslips through biotin-neutravidin interactions. Flow chambers were further blocked with $1 \mathrm{mg} \wedge \mathrm{ml}-1 \kappa$-casein. The reaction mix with purified proteins in MRB80 buffer supplemented with $20 \mu \mathrm{M}$ porcine brain tubulin, $0.5 \mu \mathrm{M}$ X-rhodamine-tubulin, $75 \mathrm{mM} \mathrm{KCl}, 1 \mathrm{mM}$ GTP, 0.2 $\mathrm{mg} \wedge \mathrm{ml}-1 \mathrm{\kappa}$-casein, $0.1 \%$ methylcellulose and oxygen scavenger $\operatorname{mix}(50 \mathrm{mM}$ glucose, $400 \mu \mathrm{g} \wedge \mathrm{ml}-1$ glucose oxidase, $200 \mu \mathrm{g} \wedge \mathrm{ml}-1$ catalase and $4 \mathrm{mM}$ DTT) was added to the flow chamber after centrifugation in an Airfuge for 5 min at $119,000 \times$ $g$. The flow chamber was sealed with vacuum grease, and dynamic MTs were imaged immediately at $30^{\circ} \mathrm{C}$ with a TIRF microscope. All tubulin products for TIRF microscopy were from Cytoskeleton. The ImageJ plugin KymoResliceWide v0.4 (https://github.com/ekatrukha/KymoResliceWide) was used for generating kymographs illustrating the life history of MT dynamics)

Protein expression and purification for Cryo-EM. Human CAMSAP1 residues 1474-1613 encompassing the CKK domain (HsCKK) were cloned into pET28a vector and expressed in BL21(DE3) cells (Stratagene) as previously described ${ }^{2}$. Cells were harvested by centrifugation and washed with Dulbecco PBS buffer (Millipore). They were sonicated in the presence of the protease inhibitor cOmplete cocktail (Roche) in lysis buffer (50 mM HEPES, $\mathrm{pH} \mathrm{8}$, supplemented with $500 \mathrm{mM} \mathrm{NaCl}$ $10 \mathrm{mM}$ imidazole, $2 \mathrm{mM} \beta$-mercaptoethanol, $0.1 \%$ bovine deoxyribonuclease I) Protein was purified by immobilized metal-affinity chromatography (IMAC) using Ni-NTA resin (Qiagen), metal-affinity chromatography (IMAC): the column was equilibrated in IMAC buffer A (50 mM HEPES, pH 8, supplemented with $500 \mathrm{mM}$ $\mathrm{NaCl}, 10 \mathrm{mM}$ imidazole, $2 \mathrm{mM} \beta$-mercaptoethanol) and the protein was eluted by IMAC buffer B (IMAC buffer A containing $400 \mathrm{mM}$ imidazole). Protein samples were subsequently loaded onto a size exclusion chromatography (SEC) HiLoad Superdex 75 16/60 column (GE Healthcare), which was equilibrated in SEC buffer (20 mM Tris-HCl, pH 7.5, supplemented with $150 \mathrm{mM} \mathrm{NaCl}$ and $1 \mathrm{mM} \mathrm{DTT}$ ) and the fractions of the main peak were pooled. Purified protein was concentrated to $\sim 20 \mathrm{mg} \wedge \mathrm{ml}-1$ in BRB20 buffer (20 mM PIPES, $2 \mathrm{mM} \mathrm{MgCl} 2,1 \mathrm{mM}$ EGTA, $1 \mathrm{mM}$ DTT, pH 6.8), snap-frozen and stored at $-80^{\circ} \mathrm{C}$.

The DNA encoding for the CKK domain of $N$. gruberi CAMSAP (NgCKK, residues 621-788; Uniprot Gene: NAEGRDRAFT_50049) was cloned into the pETbased bacterial expression vector PSPCm2, which encodes for an N-terminal $6 \mathrm{x}$ His-tag and a PreScission cleavage site using a positive selection cloning approach $^{53}$. Following protein expression in BL21 (DE3) RIPL cells (Agilent),
$\mathrm{NgCKK}$ was purified as above for HsCKK using immobilized metal-affinity chromatography (IMAC) and size exclusion chromatography (SEC). Purified protein was concentrated to $\sim 24 \mathrm{mg} \wedge \mathrm{ml}-1$ in BRB20 buffer, snap-frozen and stored at $-80^{\circ} \mathrm{C}$. Protein quality and identity were analyzed by SDS-PAGE and mass spectrometry, respectively.

tsA201 cell tubulin was purified from tsA201 cell cultures as described previously $29,54,55$. Briefly, tubulin was isolated from cell lysates via immobilized TOG1 affinity, then tubulin eluted with $0.5 \mathrm{M}$ ammonium sulfate. Tubulin was then buffer exchanged into BRB80 buffer ( $80 \mathrm{mM}$ PIPES, $2 \mathrm{mM} \mathrm{MgCl}, 1 \mathrm{mM}$ EGTA, 1 mM DTT, pH 6.8) with $10 \%$ glycerol, and $20 \mu \mathrm{M}$ GTP and flash frozen in liquid nitrogen. The tubulin was further purified by cycling ${ }^{56}$ then buffer exchanged into BRB80 with $20 \mu \mathrm{M}$ GTP and flash frozen in liquid nitrogen.

Cryo-EM sample preparation. MTs were polymerized using using $5 \mathrm{mg} / \mathrm{ml}$ tsA201 cell tubulin at $37^{\circ} \mathrm{C}$ for $45 \mathrm{~min}$ in BRB80 containing $1 \mathrm{mM}$ GTP. $1 \mathrm{mM}$ paclitaxel in DMSO was then added and MTs incubated at $37^{\circ} \mathrm{C}$ for another 45 min. Paclitaxel-stabilised MTs were used to minimise tubulin background and HsCKK-induced tubulin aggregation ${ }^{2}$, while producing roughly equal numbers of 13- and 14- protofilament MTs and having no effect on protofilament supertwist ${ }^{57}$ Stabilised MTs were left at room temperature for at least $24 \mathrm{~h}$ then diluted in BRB20 to $0.5 \mathrm{mg} \wedge \mathrm{ml}-1$ before use. Four microlitre of MTs in BRB20 were pre incubated on glow-discharged holey C-flat ${ }^{\mathrm{TM}}$ carbon EM grids (Protochips, Morrisville, NC) at room temperature for $90 \mathrm{~s}$, excess buffer manually blotted away, then $4 \mu \mathrm{l}$ of $1 \mathrm{mg} / \mathrm{ml} \mathrm{HsCKK}$ domain or NgCKK added for $45 \mathrm{~s}$. Excess buffer was again manually blotted away, followed by a final $4 \mu \mathrm{l}$ application of either HsCKK or $\mathrm{NgCKK}$ at the same concentration. Grids were then placed in a Vitrobot Mark IV (FEI Co., Hillsboro, OR) at room temperature and $80 \%$ humidity, incubated for a further $45 \mathrm{~s}$, then blotted and vitrified in liquid ethane.

Cryo-EM data collection and processing. Low dose movies were collected manually on a K2 direct electron detector (Gatan) installed on a FEI Tecnai G2 Polara operating at $300 \mathrm{kV}$ with a quantum post-column energy-filter (Gatan), operated in zero-loss imaging mode with a $20-\mathrm{eV}$ energy-selecting slit. A defocus range of $0.5-3.5 \mu \mathrm{m}$ and a calibrated final sampling of $1.39 \AA \wedge$ pixel- 1 was used with the $\mathrm{K} 2$ operating in counting mode at $5 \mathrm{e}-\wedge$ pixel- $-1 \wedge$ second -1 . The total exposure was $\sim 42 \mathrm{e}-\wedge \AA-2$ over $16 \mathrm{~s}$ at 4 frames ${ }^{\wedge}$ sec- 1 . Movie frames were aligned using Motioncorr $2^{58}$ with a patch size of 5 to generate full dose and dose-weighted sums Full dose sums were used for CTF determination in gCTF $^{59}$, then dose-weighted sums used in particle picking, processing and generation of the final reconstructions.

MTs were boxed manually in RELION's (v3.0) helical mode ${ }^{25-27}$ with a box separation distance of $82 \AA$ (roughly the MT dimer repeat distance) and further processed using a custom pipeline designed to account for the pseudo-helical nature of MTs with a seam ${ }^{28}$. The pipeline, inspired by previous pipelines ${ }^{60,61}$ developed for Spider/Frealign ${ }^{62,63}$, is based in RELION and uses accessory scripts to place refinement and classification restraints on individual MTs using prior knowledge of MT geometry. Briefly, the protofilament number of all segments within each MT was assigned according to the modal class of those segments after one iteration of 3D classification to low-pass filtered references of 11-16protofilament MTs. Only the dominant 13-protofilament and 14-protofilament classes were separately processed further. Rough alignment parameters of each MT to its corresponding low-pass filtered 13-protofilament or 14-protofilament reference were assigned. On the basis of $\varphi$ angles determined for each segment, median $\varphi$ angles were assigned to all segments in a given MT. Assigned $\varphi$ angles for each MT were checked by $3 \mathrm{D}$ classification against low-pass filtered MT references rotated and translated to represent all possible seam positions and $\alpha \beta$-tubulin registers (i.e 26 references for a 13-protofilament MT, with 13 seam positions and their counterparts translated 1 monomer along the helical axis). Rough final $\varphi$ angles were assigned according to the modal 3D class of all segments within each MT. Fine local refinement was then performed, without applied helical symmetry. Subsequently, 1 iteration each of Bayesian polishing and per-particle CTF refinement was performed in RELION v $3.0^{25}$, followed by a final round of fine local refinement with or without applied helical symmetry.

$4 \times$ binned data were used in all processing steps except the final 3D refinement ( $1 \times$ binned data) and segment averages of 7 segments along the helical axis were used for 3D classification steps. Final global resolutions are estimated from the Fourier shell correlation 0.143 cut-off of gold-standard FSCs generated by applying soft masks to the central $15 \%$ portion of the two independent half-maps along the helical axis. Reconstructions used for model building, refinement and display were sharpened (using globally determined B-factors, see Supplementary Table 1) to local resolution cut-offs determined using RELION v3.0's internal local resolution program (see Supplementary Fig. 1).

Cryo-EM model building and refinement. $\alpha 1 \mathrm{~B} / \beta \mathrm{I}+\beta \mathrm{IVb}$ tsA201 cell tubulin with bound HsCKK or NgCKK was modelled into pseudo-symmetrised density maps via iterative rounds of direct model building in $\mathrm{Coot}^{64}$ and real-space refinement applied in Phenix ${ }^{65}$. Tubulin in the starting models was constructed by fitting dimers from the GDP-EB3 13pf microtubule cryo-EM structure (PDB 3JAR ${ }^{42}$ ), into corresponding densities and applying mutations to account for sequence 
differences and incorporating taxol from the structure of tubulin-taxol zinc sheets (PDB $1 \mathrm{JFF}^{66}$ ). $\mathrm{NgCKK}$ and $\mathrm{HsCKK}$ domain starting models were created via homology modelling of the X-ray and NMR structures of CAMSAP3 CKK domain (PDBs 5LZN ${ }^{2}$ and $1 \mathrm{UGJ}$ (unpublished)) using Modeller ${ }^{67}$. Starting models of $\mathrm{HsCKK}$ or $\mathrm{NgCKK}$ domains were then fitted into density alongside tubulin and merged into single starting models constructed of six tubulin dimers and four CKK domains. Cryo-EM figures were prepared using UCSF Chimera ${ }^{68}$.

\section{Protein preparations for NMR. Human CAMSAP1 N1492A CKK (residues} 1474-1613) and mouse CAMSAP3 CKK (residues 1112-1252) were cloned into a pET28a vector as for WT CAMSAP1 CKK (above). For sample preparation of low MAS ssNMR, uniformly $\left[{ }^{13} \mathrm{C},{ }^{15} \mathrm{~N}\right]$-labeled CAMSAP1 N1492A CKK was produced in E. coli strain Rosetta 2 in M9 minimum medium supplemented with $25 \mu \mathrm{g}$ $\wedge \mathrm{ml}-1$ kanamycin and $35 \mu \mathrm{g} \wedge \mathrm{ml}-1$ chloramphenicol. Cells were induced when the $\mathrm{OD}_{600}$ reached 0.6 with $0.3 \mathrm{mM}$ IPTG at $25^{\circ} \mathrm{C}$ for $5 \mathrm{~h}$. For ${ }^{1} \mathrm{H}$ detected ssNMR experiments, uniformly $\left[{ }^{2} \mathrm{H},{ }^{13} \mathrm{C},{ }^{15} \mathrm{~N}\right]$-labeled $\mathrm{CKK}$ mutant was produced in $\mathrm{E}$. coli Rosetta 2 strain in $\mathrm{M} 9$ minimum medium that was prepared with $\mathrm{D}_{2} \mathrm{O}$, deuterated ${ }^{13} \mathrm{C}$-glucose and ${ }^{15} \mathrm{~N}_{-} \mathrm{NH}_{4} \mathrm{Cl}$. When $\mathrm{OD}_{600}$ reached $0.6,0.3 \mathrm{mM}$ IPTG was added for induction at $25^{\circ} \mathrm{C}$ for $5 \mathrm{~h}$. The proteins were purified by a ÄKTA pure system with a POROS ${ }^{\text {wn }} \mathrm{MC}$ column that was saturated with $\mathrm{Ni}^{2+}$. The column was first equilibrated with washing buffer $(50 \mathrm{mM}$ phosphate buffer, $\mathrm{pH} 8,200 \mathrm{mM} \mathrm{NaCl}, 1$ $\mathrm{mM} \beta$-mercaptoethanol and $20 \mathrm{mM}$ imidazole). Proteins were eluted with the same buffer but containing $400 \mathrm{mM}$ imidazole. Proteins were then loaded onto a SEC HiLoad Superdex 75 26/60 column (GE Healthcare) that was equilibrated with 40 $\mathrm{mM}$ phosphate buffer, $\mathrm{pH} 7$, supplemented with $150 \mathrm{mM} \mathrm{NaCl}$ and $1 \mathrm{mM}$ DTT. Subsequently, the labeled proteins were concentrated and used for ssNMR sample preparation.

To prepare CKK-MT complexes for low-speed MAS ssNMR, $20 \mathrm{mg}$ of lyophilized porcine tubulin was dissolved in BRB80 buffer to a final concentration of $2 \mathrm{mg} \wedge \mathrm{ml}-1$. Microtubule polymerization was done with $1 \mathrm{mM} \mathrm{GTP}$ and addition of $20 \mu \mathrm{M}$ paclitaxel (Sigma) for $30 \mathrm{~min}$ at $30^{\circ} \mathrm{C}$. Paclitaxel-stabilized MTs were pelleted down at $180,000 \times \mathrm{g}$ (Beckman TLA- 55 rotor) at $30^{\circ} \mathrm{C}$ for $30 \mathrm{~min}$ and resuspended in warm BRB80 buffer with $20 \mu \mathrm{M}$ paclitaxel. $\left[{ }^{13} \mathrm{C},{ }^{15} \mathrm{~N}\right]$-labeled CKK N1492A was then added to a final concentration of $65.3 \mu \mathrm{M}(4: 1 \mathrm{CKK} /$ tubulin) and incubated at $37^{\circ} \mathrm{C}$ for $30 \mathrm{~min}$. The pellet was centrifuged down at $180,000 \times g$ (Beckman TLA-55 rotor) at $30^{\circ} \mathrm{C}$ for $30 \mathrm{~min}$ and washed with $40 \mathrm{mM}$ phosphate buffer, $\mathrm{pH} \mathrm{7,} \mathrm{without} \mathrm{disturbing} \mathrm{the} \mathrm{pellet.} \mathrm{The} \mathrm{pellet} \mathrm{was} \mathrm{then} \mathrm{transferred} \mathrm{and}$ packed into a $3.2 \mathrm{~mm}$ rotor.

To prepare the CKK-MTs complexes for ${ }^{1} \mathrm{H}$ detected experiments, uniformly $\left[{ }^{2} \mathrm{H},{ }^{13} \mathrm{C},{ }^{15} \mathrm{~N}\right]$-labeled CKK was first purified and maintained in the protonated buffer overnight to allow back-exchange of amide protons. $5 \mathrm{mg}$ lyophilized porcine brain tubulin was dissolved in $2.5 \mathrm{~mL}$ BRB80 buffer to make a concentration of $2 \mathrm{mg} / \mathrm{mL}$. Tubulin was then polymerized with $1 \mathrm{mM}$ GTP and 20 $\mu \mathrm{M}$ paclitaxel for $30 \mathrm{~min}$ at $30^{\circ} \mathrm{C}$. Paclitaxel-stabilized MTs were then ultracentrifuged at $180,000 \times g$ at $30^{\circ} \mathrm{C}$ for $30 \mathrm{~min}$ and then resuspended with warm BRB80 buffer with $20 \mu \mathrm{M}$ paclitaxel. The CKK domain was added to the resuspended MTs and incubated at $37^{\circ} \mathrm{C}$ for $30 \mathrm{~min}$. The CKK-MTs complexes were then ultracentrifuged at $180,000 \times g$ at $30^{\circ} \mathrm{C}$ for $30 \mathrm{~min}$. Finally, the pellet was washed with phosphate buffer and packed into a $1.3 \mathrm{~mm}$ NMR rotor.

For sample preparation for solution-state NMR, uniformly $\left[{ }^{13} \mathrm{C},{ }^{15} \mathrm{~N}\right]$-labeled and ${ }^{15} \mathrm{~N}$-labeled CAMSAP1 N1492A CKK and NgCKK, as well as ${ }^{15} \mathrm{~N}$-labeled CAMSAP1 and CAMSAP 3 CKK were expressed and purified in the same way as described above and supplemented with $5 \% \mathrm{D}_{2} \mathrm{O}$ for solution-state NMR measurements.

NMR experiments and data analysis. Resonance assignments of CAMSAP1 CKK $\mathrm{N} 1492 \mathrm{~A}$ and $\mathrm{NgCKK}$ were obtained from standard solution-state NMR experiments (2D HSQCs, 3D HNCA, HNCO, HNCACB, CBCA(CO)NH) on free $\left[{ }^{13} \mathrm{C}\right.$, ${ }^{15} \mathrm{~N}$ ]-labeled CKK recorded on a $600 \mathrm{MHz}$ spectroscopy (Bruker Biospin). The assignments of $\mathrm{NgCKK}$ were then transferred to the spectra recorded at $25^{\circ} \mathrm{C}$. We could assign 124 residues ( $88 \%$ assigned) and 87 residues (52\% assigned) for CAMSAP1 CKK N1492A and NgCKK, respectively. Standard MAS ssNMR experiments were conducted on a $950 \mathrm{MHz}$ standard-bore spectrometer (Bruker Biospin) equipped with a $3.2 \mathrm{~mm}$ triple-channel MAS HCN probe. The experiments include $2 \mathrm{D}{ }^{13} \mathrm{C}-{ }^{13} \mathrm{C}$ proton-driven spin-diffusion (PDSD) ${ }^{69,70}$ and NCA experiments $^{71}$ (set temperature $260 \mathrm{~K}$, MAS rate $14 \mathrm{kHz}$ ). The spin-diffusion mixing time was set to $30 \mathrm{~ms}$, and a SPECIFIC-CP 72 transfer time of $2.2 \mathrm{~ms}$ was employed for the NCA experiment. Fast MAS, ${ }^{1} \mathrm{H}$ detected, ssNMR experiments were performed on a $800 \mathrm{MHz}$ wide-bore spectrometer (Bruker Biospin) equipped with a $1.3 \mathrm{~mm}$ triple-channel MAS HXY probe. The experiments included 2D NH and $3 \mathrm{D} \mathrm{CANH}{ }^{73}$ experiments (set temperature $244 \mathrm{~K}$, MAS rate $55 \mathrm{kHz}$ ). ssNMR MT samples were stable over time as confirmed by comparing ssNMR spectra at standard and fast MAS and by conducting negative stain EM experiments before and after ssNMR measurements.

CPMG relaxation dispersion ${ }^{32}$ and CEST measurements ${ }^{33}$ were based on the $2 \mathrm{D}{ }^{1} \mathrm{H}-{ }^{15} \mathrm{~N}$ HSQC spectra and were recorded as pseudo $3 \mathrm{D}$ on the ${ }^{15} \mathrm{~N}$-labeled CAMSAP1 N1492A CKK, CAMSAP1 CKK (only CPMG were recorded), CAMSAP3 CKK and NgCKK. The acquisition times in each 2D plane are $66 \mathrm{~ms}$ for
${ }^{1} \mathrm{H}$ (direct dimension) and $48.6 \mathrm{~ms}$ for ${ }^{15} \mathrm{~N}$ (indirect dimension). CPMG relaxation dispersion experiments were conducted with temperature compensation and single scan interleaved. The data were measured at CPMG fields of 50, 150, 250, 350, 450, $550,650,750,850,950,1050,1150,1250,1400$ and $1500 \mathrm{~Hz}$, which all applied for a constant transverse relaxation time of $40 \mathrm{~ms}$. The saturation during CEST experiments were carried out with a $400 \mathrm{~ms}$ pulse of $15 \mathrm{~Hz}$ radio frequency field strength on ${ }^{15} \mathrm{~N}$. The saturation offsets ranged between 8200 and $6325 \mathrm{~Hz}$ with a spacing of $25 \mathrm{~Hz}$ on ${ }^{15} \mathrm{~N}$.

The difference of chemical-shift values between the free- and bound-state CKK on both ${ }^{1} \mathrm{H}$ and ${ }^{15} \mathrm{~N}$ dimensions were first measured in ppm and then combined as

$$
\sqrt{(\Delta N * 0.15)^{2}+\Delta H^{2}} .
$$

The signal linewidth in ${ }^{1} \mathrm{H}$ and ${ }^{15} \mathrm{~N}$ dimensions were determined to amount to 0.1 and $0.6 \mathrm{ppm}$, respectively.

CMPG data were processed as follows: For each residue, the standard deviation and the average of signal intensities with different CPMG frequencies were calculated. The ratio of these two values was plotted for every residue on the structures.

For determining chemical-shift changes between free and MT-bound CAMSAP1 CKK and CAMSAP1 CKK N1492A, we transferred solution-state NMR shifts obtained on the free variants to ssNMR experiments on the complexes assuming spectral proximity in all three independent dimensions ( $\mathrm{HN}, \mathrm{N}, \mathrm{Ca}$ ). With this strategy, we were able to transfer 48 backbone assignments as demonstrated in Supplementary Fig. 6b.

Reporting summary. Further information on research design is available in the Nature Research Reporting Summary linked to this article.

\section{Data availability}

The 13-protofilament and 14-protofilament $\mathrm{HsCKK}$ and $\mathrm{NgCKK}-\mathrm{MT}$ models along with their corresponding electron density maps are deposited in the PDB. The PDB codes are as follows: 13-protofilament HsCKK-MT, PDB: 6QUS [https://www.rcsb.org/structure/ 6QUS], 14-protofilament HsCKK-MT, PDB: 6QVJ [https://www.rcsb.org/structure/ 6QVJ], 13-protofilament NgCKK-MT, PDB: 6QUY [https://www.rcsb.org/structure/ 6QUY], 14-protofilament NgCKK-MT, PDB: 6QVE [https://www.rcsb.org/structure/ 6QVE]. The EMDB codes ( $\mathrm{C} 1$ reconstruction and symmetrised asymmetric unit) are as follows: 13-protofilament HsCKK-MT, EMDB-4643, 14-protofilament HsCKK-MT, EMDB-4654, 13-protofilament NgCKK-MT, EMDB-4644,14-protofilament NgCKKMT, EMDB-4650. The source data underlying Figs $2 e, 4 b, 5 a-c$, Supplementary Figs $6 e-$ $\mathrm{h}, 7 \mathrm{a}$ are provided as a Source Data file. Other data are available from the corresponding authors upon reasonable request.

\section{Code availability}

The ImageJ plugin KymoResliceWide v0.4 (https://github.com/ekatrukha/ KymoResliceWide) was used for generating kymographs illustrating the life history of MT dynamics. The cryo-EM image-processing scripts for use with RELION and user instructions are available at https://github.com/moores-lab/MiRP.

Received: 13 March 2019; Accepted: 23 October 2019; Published online: 20 November 2019

\section{References}

1. Akhmanova, A. \& Steinmetz, M. O. Control of microtubule organization and dynamics: two ends in the limelight. Nat. Rev. Mol. Cell Biol. 16, 711-726 (2015).

2. Atherton, J. et al. A structural model for microtubule minus-end recognition and protection by CAMSAP proteins. Nat. Struct. Mol. Biol. 24, 931-943 (2017).

3. Atherton, J., Stouffer, M., Francis, F. \& Moores, C. A. Microtubule architecture in vitro and in cells revealed by cryo-electron tomography. Acta Crystallogr. D. Struct. Biol. 74, 572-584 (2018).

4. McIntosh, J. R. et al. Microtubules grow by the addition of bent guanosine triphosphate tubulin to the tips of curved protofilaments. J. Cell. Biol. 217, 2691-2708 (2018).

5. Mandelkow, E. M., Mandelkow, E. \& Milligan, R. A. Microtubule dynamics and microtubule caps: a time-resolved cryo-electron microscopy study. J. Cell. Biol. 114, 977-991 (1991).

6. Brouhard, G. J. \& Rice, L. M. Microtubule dynamics: an interplay of biochemistry and mechanics. Nat. Rev. Mol. Cell Biol. 19, 451-463 (2018)

7. Chretien, D., Fuller, S. D. \& Karsenti, E. Structure of growing microtubule ends: two-dimensional sheets close into tubes at variable rates. J. Cell. Biol. 129, 1311-1328 (1995).

8. Martin, M. \& Akhmanova, A. Coming into focus: mechanisms of microtubule minus-end organization. Trends Cell Biol. 28, 574-588 (2018). 
9. Akhmanova, A. \& Hoogenraad, C. C. Microtubule minus-end-targeting proteins. Curr. Biol. 25, R162-R171 (2015).

10. Pongrakhananon, V. et al. CAMSAP3 maintains neuronal polarity through regulation of microtubule stability. Proc. Natl Acad. Sci. USA 115, 9750-9755 (2018).

11. Toya, M. et al. CAMSAP3 orients the apical-to-basal polarity of microtubule arrays in epithelial cells. Proc. Natl Acad. Sci. USA 113, 332-337 (2016).

12. Yau, K. W. et al. Microtubule minus-end binding protein CAMSAP2 controls axon specification and dendrite development. Neuron 82, 1058-1073 (2014).

13. Noordstra, I. et al. Control of apico-basal epithelial polarity by the microtubule minus-end-binding protein CAMSAP3 and spectraplakin ACF7. J. Cell. Sci. 129, 4278-4288 (2016).

14. Chuang, M. et al. The microtubule minus-end-binding protein patronin/ PTRN-1 is required for axon regeneration in C. elegans. Cell Rep. 9, 874-883 (2014).

15. Wang, H., Brust-Mascher, I., Civelekoglu-Scholey, G. \& Scholey, J. M. Patronin mediates a switch from kinesin-13-dependent poleward flux to anaphase B spindle elongation. J. Cell. Biol. 203, 35-46 (2013).

16. Derivery, E. et al. Polarized endosome dynamics by spindle asymmetry during asymmetric cell division. Nature 528, 280-285 (2015).

17. Khanal, I., Elbediwy, A., Diaz de la Loza, M. D. C., Fletcher, G. C. \& Thompson, B. J. Correction: Shot and Patronin polarise microtubules to direct membrane traffic and biogenesis of microvilli in epithelia. J. Cell Sci. 129, 2651-2659 (2017).

18. Wang, S. et al. NOCA-1 functions with gamma-tubulin and in parallel to Patronin to assemble non-centrosomal microtubule arrays in C. elegans. eLife 4, e08649 (2015)

19. Richardson, C. E. et al. PTRN-1, a microtubule minus end-binding CAMSAP homolog, promotes microtubule function in Caenorhabditis elegans neurons. eLife 3, e01498 (2014).

20. Marcette, J. D., Chen, J. J. \& Nonet, M. L. The Caenorhabditis elegans microtubule minus-end binding homolog PTRN-1 stabilizes synapses and neurites. eLife 3, e01637 (2014)

21. Jiang, K. et al. Microtubule minus-end stabilization by polymerization-driven CAMSAP deposition. Dev. Cell. 28, 295-309 (2014).

22. Hendershott, M. C. \& Vale, R. D. Regulation of microtubule minus-end dynamics by CAMSAPs and Patronin. Proc. Natl Acad. Sci. USA 111, 5860-5865 (2014).

23. Goodwin, S. S. \& Vale, R. D. Patronin regulates the microtubule network by protecting microtubule minus ends. Cell 143, 263-274 (2010).

24. Baines, A. J. et al. The CKK domain (DUF1781) binds microtubules and defines the CAMSAP/ssp4 family of animal proteins. Mol. Biol. Evol. 26, 2005-2014 (2009).

25. Zivanov, J. et al. New tools for automated high-resolution cryo-EM structure determination in RELION-3. eLife 7, e42166 (2018).

26. He, S. \& Scheres, S. H. W. Helical reconstruction in RELION. J. Struct. Biol. 198, 163-176 (2017).

27. Scheres, S. H. RELION: implementation of a Bayesian approach to cryo-EM structure determination. J. Struct. Biol. 180, 519-530 (2012).

28. Cook, A.D., Manka, S.W., Wang, S., Moores, C.A. \& Atherton, J. A microtubule RELION-based pipeline for cryo-EM image processing. J. Struct. Biol. https://doi.org/10.1016/j.jsb.2019.10.004 (2019).

29. Vemu, A., Atherton, J., Spector, J. O., Moores, C. A. \& Roll-Mecak, A. Tubulin isoform composition tunes microtubule dynamics. Mol. Biol. Cell. 28 3564-3572 (2017).

30. Zhang, R., LaFrance, B. \& Nogales, E. Separating the effects of nucleotide and EB binding on microtubule structure. Proc. Natl Acad. Sci. USA 115, E6191-E6200 (2018)

31. Palmer, A.G., Kroenke, C.D. \& Patrick Loria, J. in Methods in Enzymology, Vol. 339. (eds. James, T.L., Dötsch, V. \& Schmitz, U.) 204-238 (Academic Press, 2001).

32. Korzhnev, D. M. \& Kay, L. E. Probing invisible, low-populated states of protein molecules by relaxation dispersion NMR spectroscopy: an application to protein folding. Acc. Chem. Res. 41, 442-451 (2008).

33. Vallurupalli, P., Bouvignies, G. \& Kay, L. E. Studying "Invisible" excited protein states in slow exchange with a major state conformation. J. Am. Chem. Soc. 134, 8148-8161 (2012).

34. Chretien, D. \& Wade, R. H. New data on the microtubule surface lattice. Biol. Cell 71, 161-174 (1991).

35. Wade, R. H., Chretien, D. \& Job, D. Characterization of microtubule protofilament numbers. How does the surface lattice accommodate? J. Mol. Biol. 212, 775-786 (1990).

36. Gonzalez-Robles, A., Cristobal-Ramos, A. R., Gonzalez-Lazaro, M., OmanaMolina, M. \& Martinez-Palomo, A. Naegleria fowleri: light and electron microscopy study of mitosis. Exp. Parasitol. 122, 212-217 (2009).

37. Kellogg, E. H. et al. Near-atomic model of microtubule-tau interactions. Science 360, 1242-1246 (2018).
38. Zhang, R., Roostalu, J., Surrey, T. \& Nogales, E. Structural insight into TPX2stimulated microtubule assembly. eLife 6, e30959 (2017)

39. Kesten, C. et al. The companion of cellulose synthase 1 confers salt tolerance through a Tau-like mechanism in plants. Nat. Commun. 10, 857 (2019).

40. Atherton, J. et al. Conserved mechanisms of microtubule-stimulated ADP release, ATP binding, and force generation in transport kinesins. eLife $\mathbf{3}$ e03680 (2014)

41. Shang, Z. et al. High-resolution structures of kinesin on microtubules provide a basis for nucleotide-gated force-generation. eLife 3, e04686 (2014).

42. Zhang, R., Alushin, G. M., Brown, A. \& Nogales, E. Mechanistic origin of microtubule dynamic instability and its modulation by EB proteins. Cell 162, 849-859 (2015).

43. Yan, S. et al. Atomic-resolution structure of the CAP-Gly domain of dynactin on polymeric microtubules determined by magic angle spinning NMR spectroscopy. Proc. Natl Acad. Sci. USA 112, 14611-14616 (2015).

44. Hyman, A. A., Chretien, D., Arnal, I. \& Wade, R. H. Structural changes accompanying GTP hydrolysis in microtubules: information from a slowly hydrolyzable analogue guanylyl-(alpha,beta)-methylene-diphosphonate. J. Cell. Biol. 128, 117-125 (1995).

45. Vale, R. D., Coppin, C. M., Malik, F., Kull, F. J. \& Milligan, R. A. Tubulin GTP hydrolysis influences the structure, mechanical properties, and kinesin-driven transport of microtubules. J. Biol. Chem. 269, 23769-23775 (1994).

46. Manka, S. W. \& Moores, C. A. The role of tubulin-tubulin lattice contacts in the mechanism of microtubule dynamic instability. Nat. Struct. Mol. Biol. 25, 607-615 (2018).

47. Maurer, S. P., Fourniol, F. J., Bohner, G., Moores, C. A. \& Surrey, T. EBs recognize a nucleotide-dependent structural cap at growing microtubule ends. Cell 149, 371-382 (2012).

48. Guesdon, A. et al. EB1 interacts with outwardly curved and straight regions of the microtubule lattice. Nat. Cell Biol. 18, 1102-1108 (2016).

49. Maurer, S. P., Bieling, P., Cope, J., Hoenger, A. \& Surrey, T. GTPgammaS microtubules mimic the growing microtubule end structure recognized by end-binding proteins (EBs). Proc. Natl Acad. Sci. USA 108, 3988-3993 (2011).

50. Lacey, S.E., He, S., Scheres, S.H. \& Carter, A.P. Cryo-EM of dynein microtubule-binding domains shows how an axonemal dynein distorts the microtubule. elife 8, e47145 (2019).

51. Cross, R. A. Microtubule lattice plasticity. Curr. Opin. Cell Biol. 56, 88-93 (2019).

52. Mohan, R. et al. End-binding proteins sensitize microtubules to the action of microtubule-targeting agents. Proc. Natl Acad. Sci. USA 110, 8900-8905 (2013).

53. Olieric, N. et al. Automated seamless DNA co-transformation cloning with direct expression vectors applying positive or negative insert selection. BMC Biotechnol. 10, 56 (2010).

54. Vemu, A., Garnham, C. P., Lee, D. Y. \& Roll-Mecak, A. Generation of differentially modified microtubules using in vitro enzymatic approaches. Methods Enzymol. 540, 149-166 (2014).

55. Widlund, P. O. et al. One-step purification of assembly-competent tubulin from diverse eukaryotic sources. Mol. Biol. Cell. 23, 4393-4401 (2012).

56. Castoldi, M. \& Popov, A. V. Purification of brain tubulin through two cycles of polymerization-depolymerization in a high-molarity buffer. Protein Expr. Purif. 32, 83-88 (2003)

57. Manka, S. W. \& Moores, C. A. Microtubule structure by cryo-EM: snapshots of dynamic instability. Essays Biochem. 62, 737-751 (2018).

58. Zheng, S. Q. et al. MotionCor2: anisotropic correction of beam-induced motion for improved cryo-electron microscopy. Nat. Methods 14, 331-332 (2017).

59. Zhang, K. Gctf: Real-time CTF determination and correction. J. Struct. Biol. 193, 1-12 (2016).

60. Sindelar, C. V. \& Downing, K. H. The beginning of kinesin's force-generating cycle visualized at 9-A resolution. J. Cell. Biol. 177, 377-385 (2007).

61. Zhang, R. \& Nogales, E. A new protocol to accurately determine microtubule lattice seam location. J. Struct. Biol. 192, 245-254 (2015).

62. Grigorieff, N. FREALIGN: high-resolution refinement of single particle structures. J. Struct. Biol. 157, 117-125 (2007).

63. Frank, J. et al. SPIDER and WEB: processing and visualization of images in 3D electron microscopy and related fields. J. Struct. Biol. 116, 190-199 (1996)

64. Emsley, P. \& Cowtan, K. Coot: model-building tools for molecular graphics. Acta Crystallogr. D. Biol. Crystallogr. 60, 2126-2132 (2004).

65. Afonine, P. V. et al. Real-space refinement in PHENIX for cryo-EM and crystallography. Acta Crystallogr. D. Struct. Biol. 74, 531-544 (2018).

66. Lowe, J., Li, H., Downing, K. H. \& Nogales, E. Refined structure of alpha betatubulin at 3.5 A resolution. J. Mol. Biol. 313, 1045-1057 (2001)

67. Sali, A. \& Blundell, T. L. Comparative protein modelling by satisfaction of spatial restraints. J. Mol. Biol. 234, 779-815 (1993).

68. Pettersen, E. F. et al. UCSF Chimera-a visualization system for exploratory research and analysis. J. Comput. Chem. 25, 1605-1612 (2004). 
69. Szeverenyi, N. M., Sullivan, M. J. \& Maciel, G. E. Observation of spin exchange by two-dimensional fourier transform 13C cross polarization-magic-angle spinning. J. Magn. Reson. (1969) 47, 462-475 (1982).

70. Bloembergen, N. On the interaction of nuclear spins in a crystalline lattice. Physica 15, 386-426 (1949).

71. Baldus, M. Correlation experiments for assignment and structure elucidation of immobilized polypeptides under magic angle spinning. Prog. Nucl. Magn. Reson. Spectrosc. 41, 1-47 (2002).

72. Baldus, M., Petkova, A. T., Herzfeld, J. \& Griffin, R. G. Cross polarization in the tilted frame: assignment and spectral simplification in heteronuclear spin systems. Mol. Phys. 95, 1197-1207 (1998).

73. Zhou, D.H. et al. Solid-state NMR analysis of membrane proteins and protein aggregates by proton detected spectroscopy. J. Biomol. NMR 54, 291-305 (2012).

\section{Acknowledgements}

This work was funded by grants from the Medical Research Council, U.K. (MR/R000352/1), the Biotechnology and Biological Sciences Research Council, U.K. (BB/L0014211/1) and the Wellcome Trust (079605/Z/06/Z) to C.A.M., from the Dutch Science Foundation NWO ((VENI grant 722.016.002 to S.X., NWO-Groot (no. 175.010.2009.002) and TOP-PUNT (no. 718.015.001) grant to M.B.) and by uNMR-NL, National Roadmap Large-Scale NMR Facility of the Netherlands (grant 184.032.207), supported by a China Scholarship Council scholarship to C.Y., the intramural program of the National Institute of Neurological Disorder and Stroke (NINDS) and National Heart, Lung and Blood Institute (NHLBI) for A.R.M., and funding from the Swiss National Science Foundation (31003A_166608) to M.O.S.

\section{Author contributions}

J.A., Y.L., A.A., M.B., C.A.M. designed the research. M.S., A.V., A.C., S.W., A. R-M., M.O.S. supplied reagents and analytical methods. J.A., Y.L., S.X., C.Y., A. R., M.S. performed the research. J.A., Y.L., S.X., C.Y., K.J., M.S., A.A., M.B., C.A.M. analysed the data, and J.A., Y.L., M.B., C.A.M. wrote the manuscript, with contributions from all authors.

\section{Competing interests}

The authors declare no competing interests.

\section{Additional information}

Supplementary information is available for this paper at https://doi.org/10.1038/s41467019-13247-6.

Correspondence and requests for materials should be addressed to J.A., M.B. or C.A.M.

Peer review information Nature Communications thanks Gregory Alushin, Loren Andreas and the other, anonymous, reviewer(s) for their contribution to the peer review of this work.

Reprints and permission information is available at http://www.nature.com/reprints

Publisher's note Springer Nature remains neutral with regard to jurisdictional claims in published maps and institutional affiliations.

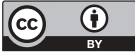

Open Access This article is licensed under a Creative Commons Attribution 4.0 International License, which permits use, sharing, adaptation, distribution and reproduction in any medium or format, as long as you give appropriate credit to the original author(s) and the source, provide a link to the Creative Commons license, and indicate if changes were made. The images or other third party material in this article are included in the article's Creative Commons license, unless indicated otherwise in a credit line to the material. If material is not included in the article's Creative Commons license and your intended use is not permitted by statutory regulation or exceeds the permitted use, you will need to obtain permission directly from the copyright holder. To view a copy of this license, visit http://creativecommons.org/ licenses/by/4.0/.

(C) The Author(s) 2019 